This article is published with open access at Springerlink.com

\title{
Triangular irreducibility of congruences in quasivarieties
}

\author{
JANUSz CzELAKOWSKI
}

\begin{abstract}
Certain forms of irreducibility as well as of equational definability of relative congruences in quasivarieties are investigated. For any integer $m \geqslant 3$ and a quasivariety $\mathbf{Q}$, the notion of an $m$-triangularily meet-irreducible $\mathbf{Q}$-congruence in the algebras of $\mathbf{Q}$ is defined. In Section 2, some characterizations of finitely generated quasivarieties involving this notion are provided. Section 3 deals with quasivarieties with equationally definable $m$-triangular meets of relatively principal congruences. References to finitely based quasivarieties and varieties are discussed.
\end{abstract}

\section{Preliminary remarks. Basic properties of quasivarieties}

Quasi-identities. Let $\tau$ be a fixed algebraic signature (a type) and let $\boldsymbol{L}_{\tau}$ be the corresponding first-order language with equality $\approx \operatorname{Var}=\left\{v_{n}: n \in \omega\right\}$ is the set of individual variables of $\boldsymbol{L}_{\tau}$. $\boldsymbol{T} \boldsymbol{e}_{\tau}$ is the algebra of terms of $\boldsymbol{L}_{\tau}$ and $E q(\tau)$ is the set of equations of $\boldsymbol{L}_{\tau}$.

A quasi-equation is a formula of the form

$$
\alpha_{1} \approx \beta_{1} \wedge \cdots \wedge \alpha_{n} \approx \beta_{n} \rightarrow \alpha \approx \beta
$$

$n=0$ is possible, so every equation qualifies as a quasi-equation.

A universally quantified quasi-equation is called a quasi-identity. As is customary, the universal quantifiers in quasi-identities are usually not explicitly written.

Any class of algebras defined by a set of quasi-identities is called a quasivariety. If $\mathbf{Q}$ is a quasivariety, then any set $\Gamma$ of quasi-identities $\operatorname{defining} \mathbf{Q}$ is called a base for $\mathbf{Q}$; we then write $\mathbf{Q}=\operatorname{Mod}(\Gamma)$.

If $\mathbf{K}$ is a class of algebras, then $\boldsymbol{Q} \boldsymbol{v}(\mathbf{K})$ is the smallest quasivariety containing $\mathbf{K}$; the class $\mathbf{K}$ is then said to generate the quasivariety $\boldsymbol{Q v}(\mathbf{K}) .(\boldsymbol{V a}(\mathbf{K})$ is the variety generated by $\mathbf{K}$.) By a result of A. Mal'cev, $\boldsymbol{Q} \boldsymbol{v}(\mathbf{K})=\boldsymbol{S} \boldsymbol{P} \boldsymbol{P}_{\boldsymbol{u}}(\mathbf{K})$ for any class $\mathbf{K}$. $\boldsymbol{S}, \boldsymbol{P}$, and $\boldsymbol{P}_{\boldsymbol{u}}$, respectively, denote the operations of forming subalgebras, direct products, and ultraproducts. (The class operations $\boldsymbol{S}, \boldsymbol{P}$, and $\boldsymbol{P}_{\boldsymbol{u}}$ are interpreted in the inclusive sense, so for example $\boldsymbol{P}_{\boldsymbol{u}}(\mathbf{K})$ is the class of all algebras isomorphic to an ultraproduct of a system of algebras from $\mathbf{K}$.)

Presented by K. Kearnes.

Received October 26, 2011; accepted in final form July 23, 2013.

2010 Mathematics Subject Classification: Primary: 08A30; Secondary: 08B05, 08B26, $08 \mathrm{C} 15$.

Key words and phrases: quasivariety, relative congruence, $m$-triangularily meetirreducible relative congruence, quasivariety with equationally definable $m$-triangular meets of (relatively) principal congruences, finitely generated quasivariety. 
A quasivariety $\mathbf{Q}$ is finitely generated if $\mathbf{Q}=\boldsymbol{Q} \boldsymbol{v}(\mathbf{K})$ for a finite set $\mathbf{K}$ of finite algebras. In this case, $\boldsymbol{Q v}(\mathbf{K})=\boldsymbol{S P}(\mathbf{K})$.

Relative congruences. Let $R$ be a binary relation defined on an algebra $\boldsymbol{A}$. $R$ is closed under the quasi-equation (1.1) if for any $a \in A^{k}, R$ contains the pair $\left\langle\alpha^{\boldsymbol{A}}(\underline{a}), \beta^{\boldsymbol{A}}(\underline{a})\right\rangle$ whenever it contains the pairs $\left\langle\alpha_{i}^{\boldsymbol{A}}(\underline{a}), \beta_{i}^{\boldsymbol{A}}(\underline{a})\right\rangle$ for $i=1, \ldots, n$. (Here $k$ is the length of a sequence $\underline{x}=x_{1}, \ldots, x_{k}$, which includes every variable occurring in at least one of the terms of (1.1). Thus, $R$ is a congruence relation on $\boldsymbol{A}$ if and only if it is closed under Birkhoff's quasi-equations:

$$
\begin{gathered}
x \approx x, \\
x \approx y \rightarrow y \approx x, \\
x \approx y \wedge y \approx z \rightarrow x \approx z,
\end{gathered}
$$

and, for each operation symbol $f$ of arity $m$,

$$
x_{1} \approx y_{1} \wedge \cdots \wedge x_{m} \approx y_{m} \rightarrow f\left(x_{1}, \ldots, x_{m}\right) \approx f\left(y_{1}, \ldots, y_{m}\right) .
$$

The set of Birkhoff's quasi-equations is denoted by Birkhoff $(\tau)$.

If $\Phi$ is a congruence of $\boldsymbol{A}$, then $\Phi$ is closed under the quasi-equation (1.1) if and only if the quotient algebra $\boldsymbol{A} / \Phi$ satisfies the quasi-identity

$$
(\forall \underline{x})\left(\alpha_{1} \approx \beta_{1} \wedge \cdots \wedge \alpha_{n} \approx \beta_{n} \rightarrow \alpha \approx \beta\right) .
$$

Notation and terminology is adopted from [15]. Accordingly, let $\mathbf{Q}$ be a quasivariety of $\tau$-algebras and $\boldsymbol{A}$ an $\tau$-algebra, not necessarily in $\mathbf{Q}$. A congruence $\Phi$ on $\boldsymbol{A}$ is called a $\mathbf{Q}$-congruence if $\boldsymbol{A} / \Phi \in \mathbf{Q}$. Denote the set of $\mathbf{Q}$-congruences of $\boldsymbol{A}$ by $\boldsymbol{C o n}_{\mathrm{Q}}(\boldsymbol{A})=\{\Phi \in \boldsymbol{C o n}(\boldsymbol{A}): \boldsymbol{A} / \Phi \in \mathbf{Q}\}$. $\boldsymbol{C o n}_{\mathrm{Q}}(\boldsymbol{A})$ contains the universal congruence $\mathbf{1}_{\boldsymbol{A}}:=A^{2}$, and it contains the identity congruence $\mathbf{0}_{\boldsymbol{A}}$ (i.e., the diagonal relation on $A$ ) iff $\boldsymbol{A} \in \mathbf{Q}$.

The family $\boldsymbol{C o n}_{\mathrm{Q}}(\boldsymbol{A})$ is closed under arbitrary intersections and the union of directed sets; in other words, $\boldsymbol{C o n}_{\mathrm{Q}}(\boldsymbol{A})$ is a finitary closure system on $A^{2}$. (This also follows from the fact that $\mathbf{Q}$ is closed under subdirect products and ultraproducts.) $\boldsymbol{C o n}_{\mathrm{Q}}(\boldsymbol{A})$ therefore forms the universe of an algebraic lattice $\boldsymbol{C o n}_{\mathbf{Q}}(\boldsymbol{A})$, called the lattice of $\mathbf{Q}$-congruences $([15,4])$.

Let $\boldsymbol{A}$ be an algebra of type $\tau$ and $X \subseteq A^{2} . \Theta_{\mathbf{Q}}^{\boldsymbol{A}}(X)$ denotes the least Q-congruence of $\boldsymbol{A}$ that contains $X$. Thus,

$$
\Theta_{\mathbf{Q}}^{\boldsymbol{A}}(X)=\bigcap\left\{\Phi \in \boldsymbol{C o n}_{\mathbf{Q}}(\boldsymbol{A}): X \subseteq \Phi\right\} .
$$

$I d(\mathbf{Q})$ denotes the set of all identities valid in $\mathbf{Q}$.

Theorem 1.1. Let $\mathbf{Q}$ be a quasivariety of algebras of type $\tau$ and $\Gamma$ a set of quasi-identities that are not identities, such that $\mathbf{Q}=\operatorname{Mod}(\operatorname{Id}(\mathbf{Q}) \cup \Gamma)$. Then for any algebra $\boldsymbol{A} \in \mathbf{Q}$, any set $X \subseteq A^{2}$, and any $a, b \in A$,

$$
a \equiv b\left(\Theta_{\mathbf{Q}}^{\boldsymbol{A}}(X)\right)
$$

if and only if there exists a finite sequence

$$
\left\langle a_{1}, b_{1}\right\rangle, \ldots,\left\langle a_{n}, b_{n}\right\rangle
$$


of elements of $A^{2}$ such that $\left\langle a_{n}, b_{n}\right\rangle=\langle a, b\rangle$ and for every $i$ with $1 \leqslant i \leqslant n$, either $\left\langle a_{i}, b_{i}\right\rangle \in X$ or $a_{i}=b_{i}$ or there exist a set $J \subseteq\{1, \ldots, i-1\}$, a quasiequation $r_{1}(\underline{x}) \approx s_{1}(\underline{x}) \wedge \cdots \wedge r_{m}(\underline{x}) \approx s_{m}(\underline{x}) \rightarrow r(\underline{x}) \approx s(\underline{x}) \in \Gamma \cup \operatorname{Birkhoff}(\tau)$, where $\underline{x}=x_{1}, \ldots, x_{p}$, and a sequence $\underline{c}=c_{1}, \ldots, c_{p}$ of elements of $A$ such that

$$
\left\{\left\langle r_{k}(\underline{c}), s_{k}(\underline{c})\right\rangle: 1 \leqslant k \leqslant m\right\}=\left\{\left\langle a_{j}, b_{j}\right\rangle: j \in J\right\} \text { and }\langle r(\underline{c}), s(\underline{c})\rangle=\left\langle a_{i}, b_{i}\right\rangle .
$$

Proof. See e.g., [5] or [9]. See also [1, Lemma Q.2.1].

The sequence $(*)$ is called a $\mathbf{Q}$-generating sequence of the pair $\langle a, b\rangle$ from the set $X$.

Equational logics. Given a class $\mathbf{K}$ of $\tau$-algebras, we let $\mathbf{K}^{e q \vDash}$ denote the consequence operation on the set of $\tau$-equations determined by $\mathbf{K}$. Thus, for $\left\{\alpha_{i} \approx \beta_{i}: i \in I\right\} \cup\{\alpha \approx \beta\} \subseteq E q(\tau)$, we have

$$
\alpha \approx \beta \in \mathbf{K}^{e q \vDash}\left(\left\{\alpha_{i} \approx \beta_{i}: i \in I\right\}\right)
$$

if and only if for every algebra $\boldsymbol{A} \in \mathbf{K}$ and every $h \in H o m\left(\boldsymbol{T e}_{\tau}, \boldsymbol{A}\right)$,

$$
h(\alpha)=h(\beta) \text { whenever } h\left(\alpha_{i}\right)=h\left(\beta_{i}\right) \text { for all } i \in I .
$$

The consequence $\mathbf{K}^{e q \vDash}$ is structural in the sense that whenever $\alpha \approx \beta \in$ $\mathbf{K}^{e q \vDash}\left(\left\{\alpha_{i} \approx \beta_{i}: i \in I\right\}\right)$, we have $e \alpha \approx e \beta \in \mathbf{K}^{e q \vDash}\left(\left\{e \alpha_{i} \approx e \beta_{i}: i \in I\right\}\right)$ for all endomorphisms $e$ of the term algebra $\mathbf{T} \boldsymbol{e}_{\tau}$. Furthermore, if $\mathbf{K}$ is closed under the formation of ultraproducts, the consequence $\mathbf{K}^{e q \vDash}$ is finitary. $\alpha \approx \beta \in$ $\mathbf{K}^{e q \vDash}(\varnothing)$ means that the equation $\alpha \approx \beta$ is valid in the class $\mathbf{K}$.

There is an obvious translation of $\mathbf{K}^{e q \vDash}$ into the language of quasi-identities over $\boldsymbol{T e}_{\tau}: \alpha \approx \beta \in \mathbf{K}^{e q \vDash}\left(\left\{\alpha_{1} \approx \beta_{1}, \ldots, \alpha_{n} \approx \beta_{n}\right\}\right)$ if and only if the implication $\alpha_{1} \approx \beta_{1} \wedge \cdots \wedge \alpha_{n} \approx \beta_{n} \rightarrow \alpha \approx \beta$ is valid in $\mathbf{K}$.

Proposition 1.2. Let $\mathbf{Q}$ be a quasivariety. Suppose that

$$
\alpha \approx \beta \in \mathbf{Q}^{e q \vDash}\left(\left\{\alpha_{i} \approx \beta_{i}: i \in I\right\}\right)
$$

for some set of equations $\left\{\alpha_{i} \approx \beta_{i}: i \in I\right\}$ and an equation $\alpha \approx \beta$. Let $\boldsymbol{A} \in \mathbf{Q}$ and let $h: \boldsymbol{T e}_{\tau} \rightarrow \boldsymbol{A}$ be a homomorphism. Then

$$
\langle h(\alpha), h(\beta)\rangle \in \Theta_{\mathbf{Q}}^{\boldsymbol{A}}\left(\left\{\left\langle h\left(\alpha_{i}\right), h\left(\beta_{i}\right)\right\rangle: i \in I\right\}\right) .
$$

Proof. Put $\Phi:=\Theta_{\mathbf{Q}}^{\boldsymbol{A}}\left(\left\{\left\langle h\left(\alpha_{i}\right), h\left(\beta_{i}\right)\right\rangle: i \in I\right\}\right)$. Let $g$ be the composition of $h$ and of the canonical homomorphism from $\boldsymbol{A}$ to the Q-algebra $\boldsymbol{A} / \Phi$. As $g$ satisfies the equations $\alpha_{i} \approx \beta_{i}, i \in I$, it follows that $g(\alpha)=g(\beta)$. So $\langle h(\alpha), h(\beta)\rangle \in \Theta_{\mathbf{Q}}^{\boldsymbol{A}}\left(\left\{\left\langle h\left(\alpha_{i}\right), h\left(\beta_{i}\right)\right\rangle: i \in I\right\}\right)$.

Free algebras. Let $\mathbf{K}$ be a class of $\tau$-algebras. $\boldsymbol{F}_{\mathbf{K}}(\omega)$ denotes the free algebra in $\mathbf{K}$ freely generated by a countably infinite set of generators.

Proposition 1.3. $\boldsymbol{F}_{\mathbf{K}}(\omega)$ is isomorphic with the quotient algebra $\boldsymbol{T e}_{\tau} / \boldsymbol{\Omega}_{0}$, where $\boldsymbol{\Omega}_{0}$ is the congruence defined as follows: for any terms $\alpha, \beta$, $\alpha \equiv \beta \quad\left(\bmod \boldsymbol{\Omega}_{0}\right)$ iff $\alpha \approx \beta \in \mathbf{K}^{e q \vDash}(\varnothing)($ iff $\alpha \approx \beta$ is valid in $\mathbf{K})$. 
Proof. The congruence $\boldsymbol{\Omega}_{0}$ is invariant, i.e., for any terms $\alpha, \beta$, if $\alpha \equiv \beta$ $\left(\bmod \boldsymbol{\Omega}_{0}\right)$, then $e \alpha \equiv e \beta\left(\bmod \boldsymbol{\Omega}_{0}\right)$, for any endomorphism of the term algebra $T e_{\tau}$.

$\boldsymbol{F}_{\mathbf{K}}(\omega)$ is also free in the variety $\boldsymbol{V a}(\mathbf{K})$. We therefore have that $\boldsymbol{F}_{\mathbf{K}}(\omega)=$ $\boldsymbol{F}_{\boldsymbol{Q v}(\mathbf{K})}(\omega)=\boldsymbol{F}_{\boldsymbol{V a}(\mathbf{K})}(\omega)$.

The equivalence class of a term $\alpha$ with respect to $\boldsymbol{\Omega}_{0}$ is denoted by $[\alpha]$. We shall identify $\boldsymbol{F}_{\mathbf{K}}(\omega)$ with $\boldsymbol{T} \boldsymbol{e}_{\tau} / \boldsymbol{\Omega}_{0}$. Consequently, $\{[x]: x$ is a variable $\}$ is the set of free generators of $\boldsymbol{F}_{\mathbf{K}}(\omega)$. Since the congruence $\boldsymbol{\Omega}_{0}$ does not paste together different variables (unless $\mathbf{K}$ is trivial), the free generators of $\boldsymbol{F}_{\mathbf{K}}(\omega)$ are often identified with individual variables.

More generally, let $\Omega$ be the mapping which to each (closed) theory $\Sigma$ of the consequence operation $\mathbf{K}^{e q \vDash}$ assigns the set of pairs

$$
\boldsymbol{\Omega}(\Sigma):=\{\langle[\alpha],[\beta]\rangle: \alpha \approx \beta \in \Sigma\} .
$$

$\boldsymbol{\Omega}(\Sigma)$ is a congruence relation in the algebra $\boldsymbol{F}_{\mathbf{K}}(\omega)$. (Note that the congruence $\boldsymbol{\Omega}_{0}$ defined as above is equal to $\left.\boldsymbol{\Omega}\left(\mathbf{K}^{e q \vDash}(\varnothing)\right)\right)$. In fact, we have the following:

Proposition 1.4. Let $\mathbf{Q}$ be a quasivariety. The mapping $\boldsymbol{\Omega}$ is an isomorphism between the lattice of closed theories of $\mathbf{Q}^{e q} \vDash$ and the congruence lattice $\operatorname{Con}_{\mathbf{Q}}\left(\boldsymbol{F}_{\mathbf{Q}}(\omega)\right)$.

The following fact is a straightforward corollary to Proposition 1.4.

Proposition 1.5. Let $\mathbf{Q}$ be a quasivariety. For any set $\Gamma$ of equations and any equation $\alpha \approx \beta$,

$$
\alpha \approx \beta \in \mathbf{Q}^{e q \vDash}(\Gamma) \quad \text { iff } \quad\langle[\alpha],[\beta]\rangle \in \Theta_{\mathbf{Q}}^{\boldsymbol{F}}(\{\langle[s],[t]\rangle: s \approx t \in \Gamma\}),
$$

where $\boldsymbol{F}:=\boldsymbol{F}_{\mathbf{Q}}(\omega)$.

More on congruences. Let $A$ and $B$ be sets and $h: A \rightarrow B$ a mapping. If $Y$ is a subset of $B^{2}$, then $h^{-1}(Y):=\left\{\langle a, b\rangle \in A^{2}:\langle h a, h b\rangle \in Y\right\}$. Similarly, if $X$ is a subset of $A^{2}$, then $h(X):=\left\{\langle h a, h b\rangle \in B^{2}:\langle a, b\rangle \in X\right\}$.

Proposition 1.6 (The Correspondence Property). Let $h: A \rightarrow \boldsymbol{B}$ be a homomorphism between arbitrary algebras $\boldsymbol{A}$ and $\boldsymbol{B}$. If $\Phi \in \boldsymbol{C o n}(\boldsymbol{A})$ and $\operatorname{ker}(h) \subseteq \Phi$, then $h^{-1} h(\Phi)=\Phi$.

Proof. (): Suppose $\langle a, b\rangle \in \Phi$. Then $\langle h a, h b\rangle \in h(\Phi)$. It follows that $\langle a, b\rangle \in$ $h^{-1} h(\Phi)$.

$(\subseteq)$ : Assume $\langle a, b\rangle \in h^{-1} h(\Phi)$. Then $\langle h a, h b\rangle \in h(\Phi)$. It follows that there are $x, y \in A$ such that $\langle h a, h b\rangle=\langle h x, h y\rangle$ and $\langle x, y\rangle \in \Phi$. As $h a=h x$ and $h b=h y$, we have $\langle a, x\rangle,\langle b, y\rangle \in \operatorname{ker}(h) \subseteq \Phi$. Hence, $\langle x, y\rangle,\langle a, x\rangle,\langle b, y\rangle \in \Phi$. This gives that $\langle a, b\rangle \in \Phi$.

Corollary 1.7. Let $\mathbf{Q}$ be a quasivariety of algebras of type $\tau$, let $h: \boldsymbol{A} \rightarrow \boldsymbol{B}$ be a homomorphism between arbitrary $\tau$-algebras, and let $\Phi \in \boldsymbol{C o n}(\boldsymbol{A})$ be a congruence such that $\operatorname{ker}(h) \subseteq \Phi$. 
(1) If $h$ is surjective and $\Phi \in \boldsymbol{C o n}_{\mathbf{Q}}(\boldsymbol{A})$, then $h(\Phi) \in \boldsymbol{C o n}_{\mathbf{Q}}(\boldsymbol{B})$.

(2) If $h(\Phi) \in \boldsymbol{C o n}_{\mathbf{Q}}(\boldsymbol{B})$, then $\Phi \in \boldsymbol{C o n}_{\mathbf{Q}}(\boldsymbol{A})$.

(3) If $h$ is surjective, then $\Phi \in \boldsymbol{C o n}_{\mathbf{Q}}(\boldsymbol{A})$ if and only if $h(\Phi) \in \boldsymbol{C o n}_{\mathbf{Q}}(\boldsymbol{B})$.

Proof. As $\operatorname{ker}(h) \subseteq \Phi$, we have that $h^{-1} h(\Phi)=\Phi$ by the Correspondence Property. It follows that the algebra $\boldsymbol{A} / \Phi$ is embeddable into $\boldsymbol{B} / h(\Phi)$. (The embedding is established by the mapping $\varphi$ which to each equivalence class $a / \Phi \in A / \Phi$ assigns the equivalence class $h a / h(\Phi)$, for each $a \in A$.)

(2): If $h(\Phi) \in \boldsymbol{C o n}_{\mathbf{Q}}(\boldsymbol{B})$, then $\boldsymbol{B} / h(\Phi) \in \mathbf{Q}$. It follows that $\boldsymbol{A} / \Phi \in \mathbf{Q}$, as $\boldsymbol{A} / \Phi$ is isomorphic with a subalgebra of the Q-algebra $\boldsymbol{B} / h(\Phi)$, proving (2).

(1): If $h$ is surjective, then the above mapping is an isomorphism between $\boldsymbol{A} / \Phi$ and $\boldsymbol{B} / h(\Phi)$. If $\Phi \in \boldsymbol{C o n}_{\mathbf{Q}}(\boldsymbol{A})$, then $\boldsymbol{A} / \Phi$ belongs to $\mathbf{Q}$, and hence $\boldsymbol{B} / h(\Phi)$ belongs to $\mathbf{Q}$ as well. Hence, $h(\Phi) \in \boldsymbol{C o n}_{\mathbf{Q}}(\boldsymbol{B})$, proving (1).

(3): This follows from (1) and (2).

Proposition 1.8. Let $\mathbf{Q}$ be a quasivariety of algebras of type $\tau$. Let $h: \boldsymbol{A} \rightarrow \boldsymbol{B}$ be a homomorphism between arbitrary $\tau$-algebras. Then for every set $X \subseteq A^{2}$, we have $h\left(\Theta_{\mathbf{Q}}^{\boldsymbol{A}}(X)\right) \subseteq \Theta_{\mathbf{Q}}^{\boldsymbol{B}}(h(X))$.

Proof. As $\boldsymbol{A} / h^{-1}\left(\Theta_{\mathbf{Q}}^{\boldsymbol{B}}(h(X))\right)$ is isomorphic with a subalgebra of the algebra $\boldsymbol{B} /\left(\Theta_{\mathbf{Q}}^{\boldsymbol{B}}(h(X))\right) \in \mathbf{Q}$, it follows that $\boldsymbol{A} / h^{-1}\left(\Theta_{\mathbf{Q}}^{\boldsymbol{B}}(h(X))\right) \in \mathbf{Q}$. Hence, $h^{-1}\left(\Theta_{\mathbf{Q}}^{\boldsymbol{B}}(h(X))\right)$ is a $\mathbf{Q}$-congruence on $\boldsymbol{A}$. Since $X \subseteq h^{-1}\left(\Theta_{\mathbf{Q}}^{\boldsymbol{B}}(h(X))\right)$, we get that $\Theta_{\mathbf{Q}}^{\boldsymbol{A}}(X) \subseteq h^{-1}\left(\Theta_{\mathbf{Q}}^{\boldsymbol{B}}(h(X))\right)$. Consequently, $h\left(\Theta_{\mathbf{Q}}^{\boldsymbol{A}}(X) \subseteq \Theta_{\mathbf{Q}}^{\boldsymbol{B}}(h(X))\right.$.

(An alternative proof of the above inclusion is based on Theorem 1.1. For let $\langle a, b\rangle \in \Theta_{\mathbf{Q}}^{\boldsymbol{A}}(X)$ and let $\left\langle a_{1}, b_{1}\right\rangle, \ldots,\left\langle a_{n}, b_{n}\right\rangle$ be a $\mathbf{Q}$-generating sequence of $\langle a, b\rangle$ from $X$ in $\boldsymbol{A}$. Then $\left\langle h a_{1}, h b_{1}\right\rangle, \ldots,\left\langle h a_{n}, h b_{n}\right\rangle$ is a Q-generating sequence of $\langle h a, h b\rangle$ from $h(X)$ in $\boldsymbol{B}$. Hence, $\langle a, b\rangle \in \Theta_{\mathbf{Q}}^{\boldsymbol{B}}(h(X))$.)

Proposition 1.9. Let $\mathbf{Q}$ be a quasivariety, $\boldsymbol{A}$ and $\boldsymbol{B}$ algebras, where $\boldsymbol{B} \in \mathbf{Q}$, and let $h: \boldsymbol{A} \rightarrow \boldsymbol{B}$ be a surjective homomorphism. Then for any set $X \subseteq A^{2}$,

$$
h\left(\Theta_{\mathbf{Q}}^{\boldsymbol{A}}(X)+\mathbf{Q} \operatorname{ker}(h)\right)=\Theta_{\mathbf{Q}}^{\boldsymbol{B}}(h(X)) .
$$

Proof. As $\boldsymbol{A} / \operatorname{ker}(h)$ is isomorphic with $\boldsymbol{B} \in \mathbf{Q}$, it follows that $\operatorname{ker}(h)$ is a $\mathbf{Q}$ congruence on $\boldsymbol{A}$. Therefore, $\Theta_{\mathbf{Q}}^{\boldsymbol{A}}(X)+_{\mathbf{Q}} \operatorname{ker}(h)$ is a well-defined $\mathbf{Q}$-congruence. Let $\Phi:=\Theta_{\mathbf{Q}}^{\boldsymbol{A}}(X)+{ }_{\mathbf{Q}} \operatorname{ker}(h)$. As $\operatorname{ker}(h) \subseteq \Phi \in \boldsymbol{C o n}_{\mathbf{Q}}(\boldsymbol{A})$, Corollary 1.7.(1) implies that $h(\Phi)$ is a $\mathbf{Q}$-congruence on $\boldsymbol{B}$. Since $X \subseteq \Phi$, we get that $h\left(\Theta_{\mathbf{Q}}^{\boldsymbol{A}}(X)+{ }_{\mathbf{Q}} \operatorname{ker}(h)\right)=h(\Phi) \supseteq \Theta_{\mathbf{Q}}^{\boldsymbol{B}}(h(X))$.

On the other hand, as $h\left(\Theta_{\mathbf{Q}}^{\boldsymbol{A}}(X) \subseteq \Theta_{\mathbf{Q}}^{\boldsymbol{B}}(h(X))\right.$ and $h(\operatorname{ker}(h))=\mathbf{0}_{\boldsymbol{B}}$, we get that $h\left(\Theta_{\mathbf{Q}}^{\boldsymbol{A}}(X)+{ }_{\mathbf{Q}} \operatorname{ker}(h)\right)=h\left(\Theta_{\mathbf{Q}}^{\boldsymbol{A}}(X \cup \operatorname{ker}(h)) \subseteq \Theta_{\mathbf{Q}}^{\boldsymbol{B}}(h(X)) \cup h(\operatorname{ker}(h))\right)=$ $\Theta_{\mathbf{Q}}^{\boldsymbol{B}}(h(X))$, by Proposition 1.8.

Corollary 1.10. Let $\mathbf{Q}$ be a quasivariety, $\boldsymbol{A}, \boldsymbol{B}$ be algebras, where $\boldsymbol{B} \in \mathbf{Q}$, and let $h: \boldsymbol{A} \rightarrow \boldsymbol{B}$ be a surjective homomorphism. Then for any set $X \subseteq A^{2}$,

$$
h^{-1}\left(\Theta_{\mathbf{Q}}^{\boldsymbol{B}}(h X)\right)=\operatorname{ker}(h)+{ }_{\mathbf{Q}} \Theta_{\mathbf{Q}}^{\boldsymbol{A}}(X) .
$$

In particular, for all $a, b \in A$,

$$
h^{-1}\left(\Theta_{\mathbf{Q}}^{\boldsymbol{B}}(h a, h b)\right)=\operatorname{ker}(h)+_{\mathbf{Q}} \Theta_{\mathbf{Q}}^{\boldsymbol{A}}(a, b) .
$$


Proof. Suppose $X \subseteq A^{2}$. Then $\Theta_{\mathbf{Q}}^{\boldsymbol{B}}(h X)=h\left(\operatorname{ker}(h)+_{\mathbf{Q}} \Theta_{\mathbf{Q}}^{\boldsymbol{A}}(X)\right)$ by the above proposition. It follows from the Correspondence Property that

$$
h^{-1}\left(\Theta_{\mathbf{Q}}^{\boldsymbol{B}}(h X)\right)=h^{-1} h\left(\operatorname{ker}(h)+_{\mathbf{Q}} \Theta_{\mathbf{Q}}^{\boldsymbol{A}}(X)\right)=\operatorname{ker}(h)+{ }_{\mathbf{Q}} \Theta_{\mathbf{Q}}^{\boldsymbol{A}}(X) .
$$

\section{Triangular irreducibility}

Let $\mathbf{Q}$ be a quasivariety, $\boldsymbol{A} \in \mathbf{Q}$, and let $a_{1}, \ldots, a_{m}$ be a finite sequence of elements of $A$ (possibly with repetitions) of length $m \geqslant 3$. In what follows, we shall make use of the following triangular table of $\mathbf{Q}$-congruences on $\boldsymbol{A}$ :

$$
\begin{array}{ccccc}
\Theta_{\mathbf{Q}}\left(a_{1}, a_{2}\right), & \Theta_{\mathbf{Q}}\left(a_{1}, a_{3}\right), & \Theta_{\mathbf{Q}}\left(a_{1}, a_{4}\right), & \ldots & \Theta_{\mathbf{Q}}\left(a_{1}, a_{m}\right), \\
\Theta_{\mathbf{Q}}\left(a_{2}, a_{3}\right), & \Theta_{\mathbf{Q}}\left(a_{2}, a_{4}\right), & \ldots & \Theta_{\mathbf{Q}}\left(a_{2}, a_{m}\right), \\
& & & \vdots \\
& \Theta_{\mathbf{Q}}\left(a_{i}, a_{i}+1\right), & \ldots & \Theta_{\mathbf{Q}}\left(a_{i}, a_{m}\right) \\
& & & \vdots \\
& & & & \\
& & & &
\end{array}
$$

This table contains $m(m-1) / 2$ elements. It is called the triangular table of relatively principal congruences corresponding to the sequence $a_{1}, \ldots, a_{m}$.

The $\mathbf{Q}$-congruence $\bigcap_{1 \leqslant i<j \leqslant m} \Theta_{\mathbf{Q}}^{\boldsymbol{A}}\left(a_{i}, a_{j}\right)$, being the intersection of the above congruences, will be also called the triangular intersection.

Definition 2.1. Let $m \geqslant 3$ be a natural number. Let $\mathbf{Q}$ be a quasivariety, $\boldsymbol{A} \in \mathbf{Q}$, and $\Phi \in \boldsymbol{C o n}_{\mathbf{Q}}(\boldsymbol{A})$. The congruence $\Phi$ is said to be $m$-triangularily irreducible in the lattice $\boldsymbol{C o n}_{\mathbf{Q}}(\boldsymbol{A})$ if, for every sequence $a_{1}, \ldots, a_{m}$ of elements of $A$ (possibly with repetitions) of length $m$, if $\bigcap_{1 \leqslant i<j \leqslant m}\left(\Theta_{\mathbf{Q}}^{\boldsymbol{A}}\left(a_{i}, a_{j}\right)+{ }_{\mathbf{Q}} \Phi\right)=$ $\Phi$, then $a_{i} \equiv a_{j}(\Phi)$ for some $i$ and $j$ with $1 \leqslant i<j \leqslant m$.

In particular, the congruence $\mathbf{0}_{\boldsymbol{A}}$ is $m$-triangularily irreducible in the lattice $\boldsymbol{C o n}_{\mathbf{Q}}(\boldsymbol{A})$ iff for every sequence $a_{1}, \ldots, a_{m}$ of elements of $A$ of length $m$, if $\bigcap_{1 \leqslant i<j \leqslant m} \Theta_{\mathbf{Q}}^{\boldsymbol{A}}\left(a_{i}, a_{j}\right)=\mathbf{0}_{\boldsymbol{A}}$, then $a_{i}=a_{j}$ for some $i$ and $j$ with $1 \leqslant i<j \leqslant m$.

Lemma 2.2. Let $\mathbf{Q}$ be a quasivariety, $\boldsymbol{A} \in \mathbf{Q}$ and $\Phi \in \boldsymbol{C o n}_{\mathbf{Q}}(\boldsymbol{A}) . \Phi$ is $m$-triangularily irreducible in the lattice $\boldsymbol{C o n}_{\mathbf{Q}}(\boldsymbol{A})$ iff the congruence $\mathbf{0}_{\boldsymbol{A} / \Phi}$ is $m$-triangularily irreducible in the lattice $\boldsymbol{C o n}_{\mathbf{Q}}(\boldsymbol{A} / \Phi)$.

Proof. Let $h: \boldsymbol{A} \rightarrow \boldsymbol{A} / \Phi$ be the canonical homomorphism. Then $\Phi=\operatorname{ker}(h)$ and by Corollary 1.10, for all $a, b \in A$ we have

$$
h^{-1}\left(\Theta_{\mathbf{Q}}^{\boldsymbol{A} / \Phi}(a / \Phi, b / \Phi)\right)=\Phi+{ }_{\mathbf{Q}} \Theta_{\mathbf{Q}}^{\boldsymbol{A}}(a, b) .
$$


Equation (2.1) and the surjectivity of $h$ imply that for any $a_{1}, \ldots, a_{m} \in A$, the following conditions are equivalent:

$$
\begin{aligned}
\bigcap_{1 \leqslant i<j \leqslant m}\left(\Theta_{\mathbf{Q}}^{\boldsymbol{A}}\left(a_{i}, a_{j}\right)+_{\mathbf{Q}} \Phi\right) & =\Phi, \\
\bigcap_{1 \leqslant i<j \leqslant m}\left(h^{-1}\left(\Theta_{\mathbf{Q}}^{\boldsymbol{A} / \Phi}\left(a_{i} / \Phi, a_{j} / \Phi\right)\right)\right) & =h^{-1}\left(\mathbf{0}_{\boldsymbol{A} / \Phi}\right), \\
h^{-1}\left(\bigcap_{1 \leqslant i<j \leqslant m} \Theta_{\mathbf{Q}}^{\boldsymbol{A} / \Phi}\left(a_{i} / \Phi, a_{j} / \Phi\right)\right) & =h^{-1}\left(\mathbf{0}_{\boldsymbol{A} / \Phi}\right), \\
\bigcap_{1 \leqslant i<j \leqslant m} \Theta_{\mathbf{Q}}^{\boldsymbol{A} / \Phi}\left(a_{i} / \Phi, a_{j} / \Phi\right) & =\mathbf{0}_{\boldsymbol{A} / \Phi .}
\end{aligned}
$$

From these conditions we get the following statement:

$\Phi$ is $m$-triangularily irreducible in the lattice $\boldsymbol{C o n}_{\mathbf{Q}}(\boldsymbol{A})$

$$
\begin{gathered}
\Longleftrightarrow\left(\forall a_{1}, \ldots, a_{m} \in A\right)\left(\bigcap_{1 \leqslant i<j \leqslant m}\left(\Theta_{\mathbf{Q}}^{\boldsymbol{A}}\left(a_{i}, a_{j}\right)+\mathbf{Q} \Phi\right)=\Phi\right. \\
\left.\Longrightarrow a_{i} \equiv a_{j}(\Phi) \text { for some } i \text { and } j\right) \\
\Longleftrightarrow\left(\forall a_{1}, \ldots, a_{m} \in A\right)\left(\bigcap_{1 \leqslant i<j \leqslant m} \Theta_{\mathbf{Q}}^{\boldsymbol{A} / \Phi}\left(a_{i} / \Phi, a_{j} / \Phi\right)=\mathbf{0}_{\boldsymbol{A} / \Phi}\right. \\
\left.\Longleftrightarrow a_{i} \equiv a_{j}(\Phi) \text { for some } i \text { and } j\right) \\
\Longrightarrow\left(\forall a_{1}, \ldots, a_{m} \in A\right)\left(\bigcap_{1 \leqslant i<j \leqslant m} \Theta_{\mathbf{Q}}^{\boldsymbol{A} / \Phi}\left(a_{i} / \Phi, a_{j} / \Phi\right)=\mathbf{0}_{\boldsymbol{A} / \Phi}\right. \\
\left.\Longrightarrow a_{i} / \Phi=a_{j} / \Phi \text { for some } i \text { and } j\right)
\end{gathered}
$$

$\Longleftrightarrow \mathbf{0}_{\boldsymbol{A} / \Phi}$ is $m$-triangularily irreducible in the lattice $\boldsymbol{C o n}_{\mathbf{Q}}(\boldsymbol{A} / \Phi)$.

$\mathrm{Q}_{\mathrm{RFSI}}$ is the class of non-trivial relatively finitely subdirectly irreducible algebras of $\mathbf{Q}$. An algebra $\boldsymbol{A} \in \mathbf{Q}$ is $m$-triangularily irreducible in $\mathbf{Q}$ if $\mathbf{0}_{\boldsymbol{A}}$ is $m$-triangularily irreducible in the lattice $\boldsymbol{C o n}_{\mathbf{Q}}(\boldsymbol{A}) \cdot \mathbf{Q}_{m-T R I}$ is the class of all algebras $m$-triangularily irreducible in $\mathbf{Q}$.

It is clear that every algebra in $\mathbf{Q}$ of cardinality less than $m$ belongs to $\mathbf{Q}_{m-T R I}$.

Lemma 2.3. Let $\mathbf{Q}$ be a quasivariety. Then $\mathbf{Q}_{\mathrm{RFSI}} \subseteq \mathbf{Q}_{m-T R I}$ for all $m \geqslant 3$.

Proof. This is immediate.

The following theorem provides a useful characterization of the class $\mathbf{Q}_{m-T R I}$ for all $m>3$.

Theorem 2.4 (K. Kearnes). Suppose $m>3$ and let $\boldsymbol{A}$ be an algebra in $\mathbf{Q}$. Then $\boldsymbol{A} \in \mathbf{Q}_{m \text {-TRI }}$ iff $|A|<m$ or $\boldsymbol{A} \in \mathbf{Q}_{\mathrm{RFSI}}$.

The theorem states that for any $m>3$, the class $\mathbf{Q}_{m \text {-TRI }}$ is the union of the class of $\mathbf{Q}$-algebras of size less than $m$ and of the class of all relatively finitely meet irreducible algebras of $\mathbf{Q}$. The theorem is false for $m=3$. (The implication $(\Leftarrow)$ holds for $m=3$ but the reverse implication does not.) A suitable 
example was produced by Keith Kearnes. Let $\boldsymbol{A}$ be the reduct of the Abelian group $\boldsymbol{Z}_{4}$ to the operation $f(x, y):=2 x-y$. Let $\mathbf{V}$ be the variety $\boldsymbol{H} \boldsymbol{S P}(\boldsymbol{A})$. $\boldsymbol{A}$ has 4 elements, $\boldsymbol{A}$ belongs to $\mathbf{V}_{3-T R I}$ but $\boldsymbol{A}$ is not (finitely) subdirectly irreducible (in $\mathbf{V}$ ).

Proof. Fix $m>3$.

$(\Leftarrow)$ : If $\boldsymbol{A} \in \mathbf{Q}_{\mathrm{RFSI}}$, then $\boldsymbol{A} \in \mathbf{Q}_{m \text {-TRI }}$ by Lemma 2.3. If the size of $\boldsymbol{A}$ is less than $m$, then trivially $\boldsymbol{A} \in \mathbf{Q}_{m-T R I}$.

$(\Rightarrow)$ : Assume $\boldsymbol{A} \in \mathbf{Q}_{m \text {-TRI. }}$. It suffices to show that if $|A| \geqslant m$, then $\boldsymbol{A} \in \mathbf{Q}_{\mathrm{RFSI}}$. Suppose $\boldsymbol{A} \notin \mathrm{Q}_{\mathrm{RFSI}}$. There exist $a, b, c, d \in A$ such that $\Theta_{\mathbf{Q}}^{\boldsymbol{A}}(a, b) \cap \Theta_{\mathbf{Q}}^{\boldsymbol{A}}(c, d)=\mathbf{0}_{\boldsymbol{A}}$ and $a \neq b, c \neq d$. The set $\{a, b, c, d\}$ has at least 3 elements. As $|A| \geqslant m>3$, we can enlarge $\{a, b, c, d\}$ to a subset $X=$ $\left\{a_{1}, \ldots, a_{m}\right\} \subseteq A$ of size exactly $m$ so that $\bigcap_{1 \leqslant i<j \leqslant m} \Theta_{\mathbf{Q}}^{\boldsymbol{A}}\left(a_{i}, a_{j}\right)=\mathbf{0}_{\boldsymbol{A}}$ and no two elements of $X$ are equal. This contradicts the fact that $\boldsymbol{A} \in \mathbf{Q}_{m \text {-TRI. }}$.

It follows from Lemma 2.3 that for each $m \geqslant 3$, any quasivariety $\mathbf{Q}$ has enough $m$-triangularily irreducible algebras in the sense that every algebra of $\mathbf{Q}$ is isomorphic with a subdirect product of a family of algebras from the class $\mathbf{Q}_{m-T R I}$.

The following theorem characterizes finitely generated quasivarieties via triangular intersections.

Theorem 2.5. Let $\mathbf{Q}$ be an arbitrary quasivariety and $m \geqslant 3$ a positive integer. The following conditions are equivalent:

(1) $\mathbf{Q}$ is generated by a finite class of algebras each of which has at most $m-1$ elements.

(2) For every algebra $\boldsymbol{A} \in \mathbf{Q}$ and for any sequence $a_{1}, \ldots, a_{m}$ of elements of $A$ of length $m$ (possibly with repetitions), it is the case that

$$
\bigcap_{1 \leqslant i<j \leqslant m} \Theta_{\mathbf{Q}}^{\boldsymbol{A}}\left(a_{i}, a_{j}\right)=\mathbf{0}_{\boldsymbol{A}} .
$$

(3) For every algebra $\boldsymbol{A} \in \mathbf{Q}$, for any congruence $\Phi \in \boldsymbol{C o n}_{\mathbf{Q}}(\boldsymbol{A})$, and any sequence $a_{1}, \ldots, a_{m}$ of elements of $A$ of length $m$ (possibly with repetitions) it is the case that

$$
\bigcap_{1 \leqslant i<j \leqslant m}\left(\Phi+{ }_{\mathbf{Q}} \Theta_{\mathbf{Q}}^{\boldsymbol{A}}\left(a_{i}, a_{j}\right)\right)=\Phi .
$$

(4) For any sequence $x_{1}, \ldots, x_{m}$ of $m$ different free generators of the free algebra $\boldsymbol{F}:=\boldsymbol{F}_{\mathbf{Q}}(\omega)$ and any congruence $\Phi \in \boldsymbol{C o n}_{\mathbf{Q}}(\boldsymbol{F})$,

$$
\bigcap_{1 \leqslant i<j \leqslant m}\left(\Phi+{ }_{\mathbf{Q}} \Theta_{\mathbf{Q}}^{\boldsymbol{F}}\left(x_{i}, x_{j}\right)\right)=\Phi .
$$

Note. Condition (4) is equivalent to

(5) For any sequence $x_{1}, x_{2}, \ldots, x_{m}$ of $m$ different individual variables and any set of equations $X$,

$$
\bigcap_{1 \leqslant i<j \leqslant m} \mathbf{Q}^{e q \vDash}\left(X \cup\left\{x_{i} \approx x_{j}\right\}\right)=\mathbf{Q}^{e q \vDash}(X) .
$$


Equivalently, (5) holds for any finite set of equations $X$. But in view of the above theorem, (5) is equivalent to

(6) For any sequence $\alpha_{1}, \alpha_{2}, \ldots, \alpha_{m}$ of terms and any set of equations $X$,

$$
\bigcap_{1 \leqslant i<j \leqslant m} \mathbf{Q}^{e q \vDash}\left(X \cup\left\{\alpha_{i} \approx \alpha_{j}\right\}\right)=\mathbf{Q}^{e q \vDash}(X) .
$$

Indeed, (6) trivially implies (5). Conversely, assume (5). As (5) is equivalent to (4), the theorem implies that (3) holds. In particular, we get that (3) holds for the free algebra $\boldsymbol{F}_{\mathbf{Q}}(\omega)$. But the last condition is equivalent to (6). So (6) holds.

Proof. (1) $\Rightarrow(4)$ : Assuming (1), we prove (5). There is a finite class of algebras $\mathbf{K}$ such that each algebra in $\mathbf{K}$ is of cardinality $<m$ and $\mathbf{Q}=\boldsymbol{S P}(\mathbf{K})$. Consequently, $\mathbf{Q}^{e q \vDash}=\mathbf{K}^{e q \vDash}$. Let $X$ be a theory of $\mathbf{Q}^{e q \vDash}$. It suffices to show that $\bigcap_{1 \leqslant i<j \leqslant m} \mathbf{Q}^{e q \vDash}\left(X \cup\left\{x_{i} \approx x_{j}\right\}\right) \subseteq X$.

Let $m$ be as above and suppose that $\alpha \approx \beta \notin X$. There exists an algebra $\boldsymbol{A} \in \mathbf{K}$ and a homomorphism $v: \boldsymbol{T e}_{\tau} \rightarrow \boldsymbol{A}$ such that $v$ satisfies $X$ and $v(\alpha) \neq$ $v(\beta)$. As $|A|<m$, we have that $v\left(x_{i}\right)=v\left(x_{j}\right)$ for some $1 \leqslant i<j \leqslant m$. It follows that $\alpha \approx \beta \notin \mathbf{Q}^{e q \vDash}\left(X \cup\left\{x_{i} \approx x_{j}\right\}\right)$ by the definition of $\mathbf{Q}^{e q \vDash}$, and consequently, $\alpha \approx \beta \notin \bigcap_{1 \leqslant i<j \leqslant m} \mathbf{Q}^{e q \vDash}\left(X \cup\left\{x_{i} \approx x_{j}\right\}\right)$. So (5) holds.

$(4) \Rightarrow(2)$ : The proof of this implication is based on two claims. Assume (4).

Claim 1. For every countable algebra $\boldsymbol{A} \in \mathbf{Q}$ and for any sequence $a_{1}, \ldots, a_{m}$ of elements of $A$ of length $m$ (possibly with repetitions),

$$
\bigcap_{1 \leqslant i<j \leqslant m} \Theta_{\mathbf{Q}}^{\boldsymbol{A}}\left(a_{i}, a_{j}\right)=\mathbf{0}_{\boldsymbol{A}} .
$$

Proof of the Claim. Assume $\boldsymbol{A} \in \mathbf{Q}$ is countable and let $a_{1}, \ldots, a_{m}$ be a sequence of elements of $A$.

Let $h: \boldsymbol{F} \rightarrow \boldsymbol{A}$ be a surjective homomorphism such that $h\left(x_{i}\right)=a_{i}$ for $i=1,2, \ldots, m$. Let $\Phi$ be the kernel of $h$. $\Phi$ is a $\mathbf{Q}$-congruence of $\boldsymbol{F}$ because the quotient algebra $\boldsymbol{F} / \Phi$ is isomorphic with $\boldsymbol{A}$.

Corollary 1.10 and the surjectivity of $h$ imply the following statement:

$$
\begin{aligned}
& \bigcap_{1 \leqslant i<j \leqslant m}\left(\Phi+{ }_{\mathbf{Q}} \Theta_{\mathbf{Q}}^{\boldsymbol{F}}\left(x_{i}, x_{j}\right)\right)=\Phi \\
& \Longleftrightarrow \bigcap_{1 \leqslant i<j \leqslant m} h^{-1}\left(\Theta_{\mathbf{Q}}^{\boldsymbol{F} / \Phi}\left(x_{i} / \Phi, x_{j} / \Phi\right)\right)=\Phi \\
& \Longleftrightarrow \bigcap_{1 \leqslant i<j \leqslant m} h^{-1}\left(\Theta_{\mathbf{Q}}^{\boldsymbol{F} / \Phi}\left(x_{i} / \Phi, x_{j} / \Phi\right)\right)=h^{-1}\left(\mathbf{0}_{F / \Phi}\right) \\
& \Longleftrightarrow h^{-1}\left(\bigcap_{1 \leqslant i<j \leqslant m} \Theta_{\mathbf{Q}}^{\boldsymbol{F} / \Phi}\left(x_{i} / \Phi, x_{j} / \Phi\right)\right)=h^{-1}\left(\mathbf{0}_{F / \Phi}\right) \\
& \Longleftrightarrow h^{-1}\left(\bigcap_{1 \leqslant i<j \leqslant m} \Theta_{\mathbf{Q}}^{\boldsymbol{A}}\left(a_{i}, a_{j}\right)\right)=h^{-1}\left(\mathbf{0}_{\boldsymbol{A}}\right) \\
& \Longleftrightarrow \bigcap_{1 \leqslant i<j \leqslant m} \Theta_{\mathbf{Q}}^{\boldsymbol{A}}\left(a_{i}, a_{j}\right)=\mathbf{0}_{\boldsymbol{A}} .
\end{aligned}
$$

As the first equality holds by (4), the last one holds also, proving the claim. 
Claim 1 continues to hold for arbitrary algebras of $\mathbf{Q}$.

Claim 2. For every algebra $\boldsymbol{A} \in \mathbf{Q}$ and for any sequence $a_{1}, \ldots, a_{m}$ of elements of $A$ of length $m$ (possibly with repetitions),

$$
\bigcap_{1 \leqslant i<j \leqslant m} \Theta_{\mathbf{Q}}^{\boldsymbol{A}}\left(a_{i}, a_{j}\right)=\mathbf{0}_{\boldsymbol{A}} .
$$

Proof of the Claim. Let $\boldsymbol{A} \in \mathbf{Q}$ and let $a_{1}, \ldots, a_{m}$ be a sequence of elements of $A$. Suppose $\langle a, b\rangle \in \bigcap_{1 \leqslant i<j \leqslant m} \Theta_{\mathbf{Q}}^{\boldsymbol{A}}\left(a_{i}, a_{j}\right)$. By Theorem 1.1, for each pair $\langle i, j\rangle$ with $i<j$ there exists a finite generating sequence

$$
\left\langle c_{i j, k}, d_{i j, k}\right\rangle, \quad k=1, \ldots, n_{i j},
$$

of the pair $\langle a, b\rangle$ from the pair $\left\langle a_{i}, a_{j}\right\rangle$ in the algebra $\boldsymbol{A}$.

Let $\boldsymbol{B}$ be the subalgebra of $\boldsymbol{A}$ generated by the elements of $A$ that are involved in the definition of the sequence $(*)_{i j}$, for all pairs $i<j$. (In particular, $\boldsymbol{B}$ contains the elements that occur in the pairs $(*)_{i j}$. But $\boldsymbol{B}$ also contains elements of $A$ that are employed by the quasi-identities applied in the definition of $\left.(*)_{i j}.\right) \boldsymbol{B}$ is countable. It follows from the definition of $\boldsymbol{B}$ that $(*)_{i j}$ is also a generating sequence of $\langle a, b\rangle$ from the pair $\left\langle a_{i}, a_{j}\right\rangle$ in the algebra $\boldsymbol{B}$, for all pairs $i<j$. Hence, $\langle a, b\rangle \in \bigcap_{1 \leqslant i<j \leqslant m} \Theta_{\mathbf{Q}}^{\boldsymbol{B}}\left(a_{i}, a_{j}\right)$. By Claim $1, a=b$.

This proves (2).

$(2) \Rightarrow(3)$ : Suppose $\boldsymbol{A} \in \mathbf{Q}, \Phi \in \boldsymbol{C o n}_{\mathbf{Q}}(\boldsymbol{A}), a_{1}, \ldots, a_{m} \in A$. Let $\boldsymbol{B}:=\boldsymbol{A} / \Phi$. Let $h: \boldsymbol{A} \rightarrow \boldsymbol{B}$ be the canonical homomorphism. Hence, $\operatorname{ker}(h)=\Phi$ and $\boldsymbol{B} \in \mathbf{Q}$. Then by Corollary 1.10 ,

$$
\begin{aligned}
& \bigcap_{1 \leqslant i<j \leqslant m}(\Phi+\mathbf{Q}\left.\Theta_{\mathbf{Q}}^{\boldsymbol{A}}\left(a_{i}, a_{j}\right)\right)=\bigcap_{1 \leqslant i<j \leqslant m} h^{-1}\left(\Theta_{\mathbf{Q}}^{\boldsymbol{B}}\left(a_{i} / \Phi, a_{j} / \Phi\right)\right) \\
&=h^{-1}\left(\bigcap_{1 \leqslant i<j \leqslant m} \Theta_{\mathbf{Q}}^{\boldsymbol{B}}\left(a_{i} / \Phi, a_{j} / \Phi\right)\right)=h^{-1}\left(\mathbf{0}_{\boldsymbol{B}}\right)=\Phi .
\end{aligned}
$$

It follows that $\bigcap_{1 \leqslant i<j \leqslant m}\left(\Phi+{ }_{\mathbf{Q}} \Theta_{\mathbf{Q}}^{\boldsymbol{A}}\left(a_{i}, a_{j}\right)\right)=\Phi$.

$(3) \Rightarrow(2)$ : This is obvious.

$(2) \Rightarrow(1)$ : Assume (2). To prove (1), it suffices to show the following:

Claim 3. Every algebra in $\mathbf{Q}_{m-T R I}$ is of cardinality $<m$.

Proof of the claim. Let $\boldsymbol{A} \in \mathbf{Q}_{m \text {-TRI }}$ and suppose a contrario that there are $m$ distinct elements in $A$, say $a_{1}, \ldots, a_{m}$. Then (2) gives $\bigcap_{1 \leqslant i<j \leqslant m} \Theta_{\mathbf{Q}}^{\boldsymbol{A}}\left(a_{i}, a_{j}\right)=$ $\mathbf{0}_{\boldsymbol{A}}$. As $\boldsymbol{A} \in \mathbf{Q}_{m-T R I}$, it follows that $a_{i}=a_{j}$ for some $i$ and $j$ with $1 \leqslant i<$ $j \leqslant m$. So $\boldsymbol{A}$ has fewer than $m$ elements, a contradiction.

This concludes the proof of the theorem.

Corollary 2.6. Suppose that $\mathbf{Q}$ is a quasivariety generated by a finite class of algebras each of which has at most $m-1$ elements, where $m \geqslant 3$. For every algebra $\boldsymbol{A} \in \mathbf{Q}$, the following conditions are equivalent:

(1) $\boldsymbol{A} \in \mathbf{Q}_{m-T R I}$,

(2) $\boldsymbol{A}$ has at most $m-1$ elements. 
Proof. This is immediate. (Note that $(2) \Rightarrow(1)$ does not require $\mathbf{Q}$ to be finitely generated.)

Corollary 2.7. Suppose that $\mathbf{Q}$ is a quasivariety generated by a finite class $\mathbf{K}$ of algebras each of which has at most $m-1$ elements, where $m \geqslant 3$. If the signature of $\mathbf{Q}$ is finite, then $\mathbf{Q}_{m \text {-TRI }}$ is a finitely axiomatizable class.

Proof. In view of Corollary 2.6, $\mathbf{Q}_{m-T R I}$ is the class of all at most $(m-1)$ element algebras of $\mathbf{Q}$. As the language of $\mathbf{Q}$ has finitely operation symbols, $\mathbf{Q}_{m-T R I}$ consists of finitely many finite algebras (up to isomorphism). It follows that $\mathbf{Q}_{m-T R I}$ is axiomatized by any set of quasi-identities which axiomatizes $\mathbf{Q}$ together with a single universal sentence

$$
\left(\forall x_{1}\right)\left(\forall x_{2}\right) \cdots\left(\forall x_{m}\right) \bigvee\left\{x_{i} \approx x_{j}: 1 \leqslant i<j \leqslant m\right\}
$$

Evidently, $\mathbf{Q}_{m-T R I}$ and its complement are algebraic classes, i.e., they are closed under isomorphisms. A simple ultraproduct argument shows that both $\mathbf{Q}_{m-T R I}$ and the complement of $\mathbf{Q}_{m-T R I}$ are closed under the formation of ultraproducts. It follows by Shelah-Keisler Theorem from the theory of models that $\mathbf{Q}_{m-T R I}$ is a finitely axiomatizable class.

\section{Quasivarieties with equationally definable $m$-triangular meets of (relatively) principal congruences}

Definition 3.1. Let $m \geqslant 3$ be a natural number and $\Lambda=\Lambda\left(x_{1}, x_{2}, \ldots, x_{m}, \underline{u}\right)$ a set of equations in variables $x_{1}, x_{2}, \ldots, x_{m}$ (and possibly some parameters $\underline{u}$ ). A quasivariety $\mathbf{Q}$ is said to have equationally definable $m$-triangular meets of (relatively) principal congruences ( $m$-EDTPM, for short) with respect to $\Lambda$ if for all $\boldsymbol{A} \in \mathbf{Q}$ and for any sequence $a_{1}, \ldots, a_{m}$ of elements $A$ of length $m$,

$$
\bigcap_{1 \leqslant i<j \leqslant m} \Theta_{\mathbf{Q}}^{\boldsymbol{A}}\left(a_{i}, a_{j}\right)=\Theta_{\mathbf{Q}}^{\boldsymbol{A}}\left((\forall \underline{e}) \Lambda^{\boldsymbol{A}}\left(a_{1}, a_{2}, \ldots, a_{m}, \underline{e}\right)\right),
$$

where $\Theta_{\mathbf{Q}}^{\boldsymbol{A}}\left((\forall \underline{e}) \Lambda^{\boldsymbol{A}}\left(a_{1}, a_{2}, \ldots, a_{m}, \underline{e}\right)\right)$ is the $\mathbf{Q}$-congruence on $A$ generated by the set of pairs $\left\langle p^{A}\left(a_{1}, a_{2}, \ldots, a_{m}, \underline{e}\right), q^{A}\left(a_{1}, a_{2}, \ldots, a_{m}, \underline{e}\right)\right\rangle$ with $\underline{e}$ ranging over sequences of elements of $A$ of the length of $\underline{u}$ and $p \approx q \in \Lambda$.

$\mathrm{Q}$ is said to have $m$-EDTPM if it has $m$-EDTPM with respect to some set of equations $\Lambda\left(x_{1}, x_{2}, \ldots, x_{m}, \underline{u}\right)$.

The following result is modelled after Lemma 2.1 in [15]:

Proposition 3.2. Let $m \geqslant 3$ be a natural number and $\Lambda=\Lambda\left(x_{1}, x_{2}, \ldots, x_{m}, \underline{u}\right)$ a set of equations in variables $x_{1}, x_{2}, \ldots, x_{m}$ (and possibly some parameters $\underline{u}$ ). Let $\mathbf{Q}$ be a quasivariety. The following conditions are equivalent:

(1) $\mathbf{Q}$ has $m$-EDTPM with respect to $\Lambda$; 
(2) For all $\boldsymbol{A} \in \mathbf{Q}$ and for any sequence $a_{1}, \ldots, a_{m}$ of elements $A$ of length $m$,

$$
\begin{aligned}
\bigcap_{1 \leqslant i<j \leqslant m} \Theta_{\mathbf{Q}}^{\boldsymbol{A}}\left(a_{i}, a_{j}\right)= & \mathbf{0}_{\boldsymbol{A}} \\
& \Leftrightarrow \boldsymbol{A} \vDash(\forall \underline{u}) \wedge \Lambda\left(x_{1}, x_{2}, \ldots, x_{m}, \underline{u}\right)\left[a_{1}, a_{2}, \ldots, a_{m}\right] ;
\end{aligned}
$$

(3) $\mathbf{Q}_{m-T R I}$ satisfies the first-order sentence

$$
\begin{aligned}
\left(\forall x_{1}\right)\left(\forall x_{2}\right) \ldots\left(\forall x_{m}\right)\left((\forall \underline{u}) \wedge \Lambda\left(x_{1}, x_{2}, \ldots, x_{m}, \underline{u}\right)\right. & \\
& \left.\leftrightarrow \bigvee\left\{x_{i} \approx x_{j}: 1 \leqslant i<j \leqslant m\right\}\right)
\end{aligned}
$$

(4) For every $i, j$ with $1 \leqslant i<j \leqslant m$, $\mathbf{Q}$ satisfies the equations $\Lambda\left(x_{j} / x_{i}\right)$ and for any algebra $\boldsymbol{A} \in \mathbf{Q}$ and any sequence $a_{1}, \ldots, a_{m}$ of elements of $A$,

$$
\bigcap_{1 \leqslant i<j \leqslant m} \Theta_{\mathbf{Q}}^{\boldsymbol{A}}\left(a_{i}, a_{j}\right) \subseteq \Theta_{\mathbf{Q}}^{\boldsymbol{A}}\left((\forall \underline{e}) \Lambda^{\boldsymbol{A}}\left(a_{1}, a_{2}, \ldots, a_{m}, \underline{e}\right)\right) .
$$

Here, $\Lambda\left(x_{j} / x_{i}\right)$ results from $\Lambda\left(x_{1}, x_{2}, \ldots, x_{m}, \underline{u}\right)$ by the uniform substitution of the variable $x_{i}$ for the variable $x_{j}$. In (3), the symbol " $\leftrightarrow$ " is the equivalence connective from the first-order language associated with the signature of $\mathbf{Q}$.

Proof. $(1) \Rightarrow(2) \Rightarrow(3)$ : This is immediate.

$(3) \Rightarrow(4)$ : (3) implies that for each $i, j$ with $1 \leqslant i<j \leqslant m, \mathbf{Q}_{m-T R I}$ satisfies the equations $\Lambda\left(x_{j} / x_{i}\right)$ and hence so does $\mathbf{Q}$.

Now let $\boldsymbol{A} \in \mathbf{Q}$ and let $a_{1}, \ldots, a_{m}$ be a sequence of elements of $A$. To prove the inclusion

$$
\bigcap_{1 \leqslant i<j \leqslant m} \Theta_{\mathbf{Q}}^{\boldsymbol{A}}\left(a_{i}, a_{j}\right) \subseteq \Theta_{\mathbf{Q}}^{\boldsymbol{A}}\left((\forall \underline{e}) \Lambda^{\boldsymbol{A}}\left(a_{1}, a_{2}, \ldots, a_{m}, \underline{e}\right)\right),
$$

it suffices to show that that for every $m$-triangularily irreducible congruence $\Phi$ in the lattice $\boldsymbol{C o n}_{\mathbf{Q}}(\boldsymbol{A})$,

$$
\Phi \supseteq \Theta_{\mathbf{Q}}^{\boldsymbol{A}}\left((\forall \underline{e}) \Lambda^{\boldsymbol{A}}\left(a_{1}, a_{2}, \ldots, a_{m}, \underline{e}\right)\right) \Longrightarrow \Phi \supseteq \bigcap_{1 \leqslant i<j \leqslant m} \Theta_{\mathbf{Q}}^{\boldsymbol{A}}\left(a_{i}, a_{j}\right) .
$$

Suppose the first inclusion holds. Then

$$
\alpha\left(b_{1}, c_{1}, b_{2}, c_{2}, \ldots, b_{r}, c_{r}, \underline{e}\right) / \Phi=\beta\left(b_{1}, c_{1}, b_{2}, c_{2}, \ldots, b_{r}, c_{r}, \underline{e}\right) / \Phi
$$

for all $\alpha \approx \beta \in \Lambda$ and all sequences $\underline{e} \in A^{k}$. Thus,

$$
\boldsymbol{A} / \Phi \vDash(\forall \underline{u}) \wedge \Lambda\left(x_{1}, x_{2}, \ldots, x_{m}, \underline{u}\right)\left[a_{1}, a_{2}, \ldots, a_{m}\right] .
$$

Since $\boldsymbol{A} / \Phi \in \mathbf{Q}_{m-T R I}$, it follows from (3) that we have $a_{i} / \Phi=a_{j} / \Phi$ for some $1 \leqslant i<j \leqslant m$. So $\Theta_{\mathbf{Q}}^{\boldsymbol{A}}\left(a_{i}, a_{j}\right) \subseteq \Phi$ for some $i<j$. Consequently, $\Phi \supseteq \bigcap_{1 \leqslant i<j \leqslant m} \Theta_{\mathbf{Q}}^{\boldsymbol{A}}\left(a_{i}, a_{j}\right)$. Thus, (*) holds.

$(4) \Rightarrow(1)$ : Let $\boldsymbol{A} \in \mathbf{Q}$ and let $a_{1}, \ldots, a_{m}$ be a sequence of elements of $A$. Fix $i, j$ with $1 \leqslant i<j \leqslant m$ and let $\Phi_{0}:=\Theta_{\mathbf{Q}}^{\boldsymbol{A}}\left(a_{i}, a_{j}\right)$. The quotient algebra $\boldsymbol{A} / \Phi_{0}$ belongs to $\mathbf{Q}$. By the first conjunct of (4), we get that $\alpha\left(a_{1}, a_{2}, \ldots, a_{m}, \underline{e}\right) / \Phi_{0}=\beta\left(a_{1}, a_{2}, \ldots, a_{m}, \underline{e}\right) / \Phi_{0}$ for all $\alpha \approx \beta \in \Lambda$ and all sequences $\underline{e} \in A^{k}$. It follows that $\Theta_{\mathbf{Q}}^{\boldsymbol{A}}\left((\forall \underline{e}) \Lambda^{\boldsymbol{A}}\left(a_{1}, a_{2}, \ldots, a_{m}, \underline{e}\right)\right) \subseteq \Theta_{\mathbf{Q}}^{\boldsymbol{A}}\left(a_{i}, a_{j}\right)$. This proves the " $\supseteq$ "-inclusion of the two inclusions of (1).

As the other inclusion is assumed by (4), condition (1) follows. 
Theorem 3.3. Let $m \geqslant 3$ be a natural number. For any quasivariety $\mathbf{Q}$, the following conditions are equivalent:

(1) $\mathbf{Q}$ has m-EDTPM.

(2) For every algebra $\boldsymbol{A} \in \mathbf{Q}$, for any sequence $a_{1}, \ldots, a_{m}$ of elements of $A$ of length $m$ (possibly with repetitions), and any congruence $\Phi \in \boldsymbol{C o n}_{\mathbf{Q}}(\boldsymbol{A})$,

$$
\Phi+\mathbf{Q} \bigcap_{1 \leqslant i<j \leqslant m} \Theta_{\mathbf{Q}}^{\boldsymbol{A}}\left(a_{i}, a_{j}\right)=\bigcap_{1 \leqslant i<j \leqslant m}\left(\Phi+\mathbf{Q} \Theta_{\mathbf{Q}}^{\boldsymbol{A}}\left(a_{i}, a_{j}\right)\right) .
$$

(3) For any sequence $x_{1}, \ldots, x_{m}$ of $m$ different free generators of the free algebra $\boldsymbol{F}:=\boldsymbol{F}_{\mathbf{Q}}(\omega)$ and any congruence $\Phi \in \boldsymbol{C o n}_{\mathbf{Q}}(\boldsymbol{F})$,

$$
\Phi+\mathbf{Q} \bigcap_{1 \leqslant i<j \leqslant m} \Theta_{\mathbf{Q}}^{\boldsymbol{F}}\left(x_{i}, x_{j}\right)=\bigcap_{1 \leqslant i<j \leqslant m}\left(\Phi+\mathbf{Q} \Theta_{\mathbf{Q}}^{\boldsymbol{F}}\left(x_{i}, x_{j}\right)\right) .
$$

Note. Condition (3) of the above theorem is equivalently expressed in terms of the consequence $\mathbf{Q}^{e q \vDash}$ as

(4) $\mathbf{Q}^{e q \vDash}\left(X \cup \bigcap_{1 \leqslant i<j \leqslant n} \mathbf{Q}^{e q \vDash}\left(x_{i} \approx x_{j}\right)\right)=\bigcap_{1 \leqslant i<j \leqslant n} \mathbf{Q}^{e q \vDash}\left(X \cup\left\{x_{i} \approx x_{j}\right\}\right)$, for any set of equations $X$.

(Here, $x_{1}, x_{2}, \ldots, x_{m}$ is an arbitrary but fixed sequence of $m$ different individual variables.)

But it is easy to see that (4) is equivalent to

(5) $\mathbf{Q}^{e q \vDash}\left(X \cup \bigcap_{1 \leqslant i<j \leqslant n} \mathbf{Q}^{e q \vDash}\left(\alpha_{i} \approx \alpha_{j}\right)\right)=\bigcap_{1 \leqslant i<j \leqslant n} \mathbf{Q}^{e q \vDash}\left(X \cup\left\{\alpha_{i} \approx \alpha_{j}\right\}\right)$, for any set of equations $X$ and any sequence of terms $\alpha_{1}, \alpha_{2}, \ldots, \alpha_{m}$.

Proof. (1) $\Rightarrow(2)$ : Assume $\mathbf{Q}$ has $m$-EDTPM with respect to a set of equations $\Lambda=\Lambda\left(x_{1}, x_{2}, \ldots, x_{m}, \underline{u}\right)$. Let $\boldsymbol{A}$ be an algebra in $\mathbf{Q}, \Phi \in \boldsymbol{C o n}_{\mathbf{Q}}(\boldsymbol{A})$, and $a_{1}, \ldots, a_{m}$ a sequence of elements of $A$ of length $m$. Let $\boldsymbol{B}:=\boldsymbol{A} / \Phi$. As $\boldsymbol{B} \in \mathbf{Q}$, (1) implies that

(a) $\bigcap_{1 \leqslant i<j \leqslant m} \Theta_{\mathbf{Q}}^{\boldsymbol{B}}\left(a_{i} / \Phi, a_{j} / \Phi\right)=\Theta_{\mathbf{Q}}^{\boldsymbol{B}}\left((\forall \underline{e}) \Lambda_{\boldsymbol{B}}\left(a_{1} / \Phi, a_{2} / \Phi, \ldots, a_{m} / \Phi, \underline{e} / \Phi\right)\right)$.

Let $h: A \rightarrow \boldsymbol{B}$ be the canonical homomorphism. Then

(b) $h^{-1}\left(\Theta_{\mathbf{Q}}^{B}(a / \Phi, b / \Phi)\right)=\Phi+{ }_{\mathrm{Q}} \Theta_{\mathbf{Q}}^{\boldsymbol{A}}(a, b)$ for all $a, b \in A$.

Item (b) and the surjectivity of $h$ imply that

$$
\text { (c) } \begin{aligned}
h^{-1}\left(\bigcap_{1 \leqslant i<j \leqslant m} \Theta_{\mathbf{Q}}^{\boldsymbol{B}}\left(a_{i} / \Phi, a_{j} / \Phi\right)\right) & =\bigcap_{1 \leqslant i<j \leqslant m} h^{-1}\left(\Theta_{\mathbf{Q}}^{\boldsymbol{B}}\left(a_{i} / \Phi, a_{j} / \Phi\right)\right) \\
& =\bigcap_{1 \leqslant i<j \leqslant m}\left(\Phi+\mathbf{Q} \Theta_{\mathbf{Q}}^{\boldsymbol{A}}\left(a_{i}, a_{j}\right)\right) .
\end{aligned}
$$

On the other hand, we also get 
(d)

$$
\begin{aligned}
& h^{-1}\left(\Theta_{\mathbf{Q}}^{\boldsymbol{B}}\left((\forall \underline{e}) \Lambda^{\boldsymbol{B}}\left(a_{1} / \Phi, a_{2} / \Phi, \ldots, a_{m} / \Phi, \underline{e} / \Phi\right)\right)\right) \\
= & h^{-1}\left(\sup \left\{\Theta_{\mathbf{Q}}^{\boldsymbol{B}}\left(\Lambda^{\boldsymbol{B}}\left(a_{1} / \Phi, a_{2} / \Phi, \ldots, a_{m} / \Phi, \underline{e} / \Phi\right)\right): \underline{e} \in A^{k}\right\}\right) \\
= & \sup \left\{h^{-1}\left(\Theta_{\mathbf{Q}}^{\boldsymbol{B}}\left(\Lambda^{\boldsymbol{B}}\left(a_{1} / \Phi, a_{2} / \Phi, \ldots, a_{m} / \Phi, \underline{e} / \Phi\right)\right)\right): \underline{e} \in A^{k}\right\} \\
= & \sup \left\{\Phi+{ }_{\mathbf{Q}} \Theta_{\mathbf{Q}}^{\boldsymbol{A}}\left(\Lambda^{\boldsymbol{A}}\left(a_{1}, a_{2}, \ldots, a_{m}, \underline{e}\right)\right): \underline{e} \in A^{k}\right\} \\
= & \Phi+\mathbf{Q} \sup \left\{\Theta_{\mathbf{Q}}^{\boldsymbol{A}}\left(\Lambda^{\boldsymbol{A}}\left(a_{1}, a_{2}, \ldots, a_{r}, \underline{e}\right)\right): \underline{e} \in A^{k}\right\} \\
= & \Phi+{ }_{\mathbf{Q}} \Theta_{\mathbf{Q}}^{\boldsymbol{A}}\left((\forall \underline{e}) \Lambda^{\boldsymbol{A}}\left(a_{1}, a_{2}, \ldots, a_{m}, \underline{e}\right)\right) \\
= & \Phi+{ }_{\mathbf{Q}} \bigcap_{1 \leqslant i<j \leqslant m} \Theta_{\mathbf{Q}}^{\boldsymbol{A}}\left(a_{i}, a_{j}\right) .
\end{aligned}
$$

But in view of (a), the first congruences of (c) and (d) are identical. Consequently, the last congruences of (c) and (d) are the same. Thus,

$$
\bigcap_{1 \leqslant i<j \leqslant m}\left(\Phi+\mathbf{Q} \Theta_{\mathbf{Q}}^{\boldsymbol{A}}\left(a_{i}, a_{j}\right)\right)=\Phi+\mathbf{Q} \bigcap_{1 \leqslant i<j \leqslant m} \Theta_{\mathbf{Q}}^{\boldsymbol{A}}\left(a_{i}, a_{j}\right) .
$$

So (2) holds.

$(2) \Rightarrow(3)$ : This is immediate.

$(3) \Rightarrow(1)$ : Let $x_{1}, x_{2}, \ldots, x_{m}$ be a finite sequence of pairwise different individual variables. Let $\Lambda\left(x_{1}, x_{2}, \ldots, x_{m}, \underline{u}\right)$ be a set of equations such that

$$
\bigcap_{1 \leqslant i<j \leqslant m} \mathbf{Q}^{e q \vDash}\left(x_{i} \approx x_{j}\right)=\mathbf{Q}^{e q \vDash}\left(\Lambda\left(x_{1}, x_{2}, \ldots, x_{m}, \underline{u}\right)\right) .
$$

Passing to the algebra $\boldsymbol{F}$, we therefore have that

$$
\bigcap_{1 \leqslant i<j \leqslant m} \Theta_{\mathbf{Q}}^{\boldsymbol{F}}\left(x_{i}, x_{j}\right)=\Theta_{\mathbf{Q}}^{\boldsymbol{A}}\left(\Lambda^{\boldsymbol{F}}\left(x_{1}, x_{2}, \ldots, x_{m}, \underline{u}\right)\right) .
$$

Let $\boldsymbol{A}$ be a countably generated algebra in $\mathbf{Q}$, let $a_{1}, a_{2}, \ldots, a_{m}$ be a fixed sequence of elements of $A$. Moreover, let $\underline{e} \in A^{k}$ be an arbitrary sequence of length $k$. Let $h: \boldsymbol{F} \rightarrow \boldsymbol{A}$ be a surjective homomorphism such that $h\left(x_{1}\right)=a_{1}$, $\ldots, h\left(x_{m}\right)=a_{m}$ and $h(\underline{u})=\underline{e}$. ( $h$ is arbitrarily defined for the remaining free generators of $\boldsymbol{F}$.) Let $\Phi \in \boldsymbol{C o n}_{\mathbf{Q}}(\boldsymbol{F})$ be the relation-kernel of $h$. Assuming (3), we have

$$
\begin{aligned}
& h^{-1}\left(\bigcap_{1 \leqslant i<j \leqslant m} \Theta_{\mathbf{Q}}^{\boldsymbol{A}}\left(a_{i}, a_{j}\right)\right)=\bigcap_{1 \leqslant i<j \leqslant m} h^{-1}\left(\Theta_{\mathbf{Q}}^{\boldsymbol{A}}\left(a_{i}, a_{j}\right)\right) \\
= & \bigcap_{1 \leqslant i<j \leqslant m}\left(\Phi+\mathbf{Q} \Theta_{\mathbf{Q}}^{\boldsymbol{F}}\left(x_{i}, x_{j}\right)\right) \stackrel{(3)}{=} \Theta_{\mathbf{Q}}^{\boldsymbol{F}}\left(\Phi \cup \bigcap_{1 \leqslant i<j \leqslant m} \Theta_{\mathbf{Q}}^{\boldsymbol{F}}\left(x_{i}, x_{j}\right)\right) \\
= & \Theta_{\mathbf{Q}}^{\boldsymbol{F}}\left(\Lambda\left(x_{1}, x_{2}, \ldots, x_{m}, \underline{u}\right)\right)+\mathbf{Q} \Phi=h^{-1}\left(\Theta_{\mathbf{Q}}^{\boldsymbol{A}}\left(\Lambda\left(a_{1}, a_{2}, \ldots, a_{m}, \underline{e}\right)\right)\right) .
\end{aligned}
$$

Item (e) gives that

$$
\bigcap_{1 \leqslant i<j \leqslant m} \Theta_{\mathbf{Q}}^{\boldsymbol{A}}\left(a_{i}, a_{j}\right)=\Theta_{\mathbf{Q}}^{\boldsymbol{A}}\left(\Lambda\left(a_{1}, a_{2}, \ldots, a_{m}, \underline{e}\right)\right) \text {, for any sequence } \underline{e} \in A^{k} \text {. }
$$

It follows that

(f) For every countably generated $\boldsymbol{A} \in \mathbf{Q}$ and all $a_{1}, a_{2}, \ldots, a_{m} \in A$, we have $\bigcap_{1 \leqslant i<j \leqslant m} \Theta_{\mathbf{Q}}^{\boldsymbol{A}}\left(a_{i}, a_{j}\right)=\mathbf{0}_{\boldsymbol{A}}$ iff $\Theta_{\mathbf{Q}}^{\boldsymbol{A}}\left(\Lambda\left(a_{1}, a_{2}, \ldots, a_{m}, \underline{e}\right)\right)=\mathbf{0}_{\boldsymbol{A}}$, for all sequences $\underline{e} \in A^{k}$. 
We then observe that (f) continues to hold for algebras $\boldsymbol{A} \in \mathbf{Q}$ of arbitrary cardinality.

(g) For any algebra $\boldsymbol{A} \in \mathbf{Q}$ and any $a_{1}, a_{2}, \ldots, a_{m} \in A$,

$$
\bigcap_{1 \leqslant i<j \leqslant m} \Theta_{\mathbf{Q}}^{\boldsymbol{A}}\left(a_{i}, a_{j}\right)=\mathbf{0}_{\boldsymbol{A}} \text { iff } \Theta_{\mathbf{Q}}^{\boldsymbol{A}}\left(\Lambda\left(a_{1}, a_{2}, \ldots, a_{m}, \underline{e}\right)\right)=\mathbf{0}_{\boldsymbol{A}} \text { for all } \underline{e} \in A^{k} .
$$

Let $a_{1}, a_{2}, \ldots, a_{m} \in A$. First we assume that $\bigcap_{1 \leqslant i<j \leqslant m} \Theta_{\mathbf{Q}}^{\boldsymbol{A}}\left(a_{i}, a_{j}\right)=\mathbf{0}_{\boldsymbol{A}}$. Let $\underline{e} \in A^{k}$ be an arbitrary sequence. Then $\bigcap_{1 \leqslant i<j \leqslant m} \Theta_{\mathbf{Q}}^{\boldsymbol{A}}\left(a_{i}, a_{j}\right)=\mathbf{0}_{\boldsymbol{A}}$ implies that $\bigcap_{1 \leqslant i<j \leqslant m} \Theta_{\mathbf{Q}}^{\boldsymbol{B}}\left(a_{i}, a_{j}\right)=\mathbf{0}_{\boldsymbol{B}}$ for some countable subalgebra $\boldsymbol{B}$ of $\boldsymbol{A}$ that contains $a_{1}, a_{2}, \ldots, a_{m}$ and $\underline{e}$. Therefore, by (f), we have that $\Theta_{\mathbf{Q}}^{\boldsymbol{B}}\left(\Lambda\left(a_{1}, a_{2}, \ldots, a_{m}, \underline{e}\right)\right)=\mathbf{0}_{\boldsymbol{B}}$. It follows that $\Theta_{\mathbf{Q}}^{\boldsymbol{A}}\left(\Lambda\left(a_{1}, a_{2}, \ldots, a_{m}, \underline{e}\right)\right)=\mathbf{0}_{\boldsymbol{A}}$ because $\boldsymbol{B}$ is a subalgebra of $\boldsymbol{A}$.

Conversely, suppose that $\Theta_{\mathbf{Q}}^{\boldsymbol{A}}\left(\Lambda\left(a_{1}, a_{2}, \ldots, a_{m}, \underline{e}\right)\right)=\mathbf{0}_{\boldsymbol{A}}$, for all $\underline{e} \in A^{k}$. Let $\boldsymbol{B}$ be an arbitrary countable subalgebra of $\boldsymbol{A}$ which contains $a_{1}, a_{2}, \ldots, a_{m}$. Then $\Theta_{\mathbf{Q}}^{\boldsymbol{B}}\left(\Lambda\left(a_{1}, a_{2}, \ldots, a_{m}, \underline{e}\right)\right)=\mathbf{0}_{\boldsymbol{B}}$ for all $\underline{e} \in B^{k}$. Thus, (f) gives that

(*) $\bigcap_{1 \leqslant i<j \leqslant m} \Theta_{\mathbf{Q}}^{\boldsymbol{B}}\left(a_{i}, a_{j}\right)=\mathbf{0}_{\boldsymbol{B}}$ in every countable subalgebra $\boldsymbol{B}$ of $\boldsymbol{A}$ which contains $a_{1}, a_{2}, \ldots, a_{m}$.

We then get that

(**) $\bigcap_{1 \leqslant i<j \leqslant m} \Theta_{\mathbf{Q}}^{\boldsymbol{A}}\left(a_{i}, a_{j}\right)=\mathbf{0}_{\boldsymbol{A}}$.

Indeed, if $\langle c, d\rangle \in \bigcap_{1 \leqslant i<j \leqslant m} \Theta_{\mathbf{Q}}^{\boldsymbol{A}}\left(a_{i}, a_{j}\right)$, then Theorem 1.1 implies that there is a countable subalgebra $\boldsymbol{B}$ of $\boldsymbol{A}$ which includes $a_{1}, a_{2}, \ldots, a_{m}$ and $c, d$ such that $\langle c, d\rangle \in \bigcap_{1 \leqslant i<j \leqslant m} \Theta_{\mathbf{Q}}^{\boldsymbol{B}}\left(a_{i}, a_{j}\right)$. It follows by $(*)$ that $c=d$. So $(* *)$ holds. This proves $(\mathrm{g})$.

But (g) is equivalent to condition (2) of Proposition 3.2. It follows that $\mathbf{Q}$ has $m$-EDTPM.

The following corollary is immediate (cf. Corollary 2.6):

Corollary 3.4. Let $m \geqslant 3$ be a natural number. Let $\mathbf{Q}$ be a quasivariety with $m$-EDTPM with respect to a set of equations $\Lambda=\Lambda\left(x_{1}, x_{2}, \ldots, x_{m}, \underline{u}\right)$. Then for any algebra $\boldsymbol{A} \in \mathbf{Q}$, the following conditions are equivalent:

(1) $\boldsymbol{A} \in \mathbf{Q}_{m-T R I}$.

(2) For every sequence $a_{1}, \ldots, a_{m}$ of elements of $A$, if

$$
\Theta_{\mathbf{Q}}^{\boldsymbol{A}}\left(\Lambda\left(a_{1}, a_{2}, \ldots, a_{m}, \underline{e}\right)\right)=\mathbf{0}_{\boldsymbol{A}} \text { for all } \underline{e} \in A^{k},
$$

then $a_{i}=a_{j}$ for some $1 \leqslant i<j \leqslant m$.

Proof. (1) $\Rightarrow(2)$ : Assume (1). Let $a_{1}, \ldots, a_{m}$ be a sequence of elements of $A$ such that $\Theta_{\mathbf{Q}}^{\boldsymbol{A}}\left(\Lambda\left(a_{1}, a_{2}, \ldots, a_{m}, \underline{e}\right)\right)=\mathbf{0}_{\boldsymbol{A}}$, for all $\underline{e} \in A^{k}$. But $m$-EDTPM gives that $\Theta_{\mathbf{Q}}^{\boldsymbol{A}}\left((\forall \underline{e}) \Lambda\left(a_{1}, a_{2}, \ldots, a_{m}, \underline{e}\right)\right)=\bigcap_{1 \leqslant i<j \leqslant m} \Theta_{\mathbf{Q}}^{\boldsymbol{A}}\left(a_{i}, a_{j}\right)$. It follows that $\bigcap_{1 \leqslant i<j \leqslant m} \Theta_{\mathbf{Q}}^{\boldsymbol{A}}\left(a_{i}, a_{j}\right)=\mathbf{0}_{\boldsymbol{A}}$.As $\boldsymbol{A} \in \mathbf{Q}_{m-T R I}$, we get that $a_{i}=a_{j}$, for some $i, j$ with $1 \leqslant i<j \leqslant m$. So (2) holds.

$(2) \Rightarrow(1)$ : The proof of this implication is similar. Assume (2). We must show that $\mathbf{0}_{\boldsymbol{A}}$ is $m$-triangularily irreducible. Let $a_{1}, \ldots, a_{m}$ be a sequence 
of elements of $A$ such that $\bigcap_{1 \leqslant i<j \leqslant m} \Theta_{\mathbf{Q}}^{\boldsymbol{A}}\left(a_{i}, a_{j}\right)=\mathbf{0}_{\boldsymbol{A}}$. But by $m$-EDTPM, $\bigcap_{1 \leqslant i<j \leqslant m} \Theta_{\mathbf{Q}}^{\boldsymbol{A}}\left(a_{i}, a_{j}\right)=\Theta_{\mathbf{Q}}^{\boldsymbol{A}}\left((\forall \underline{e}) \Lambda\left(a_{1}, a_{2}, \ldots, a_{m}, \underline{e}\right)\right)$. Hence,

$$
\Theta_{\mathbf{Q}}^{\boldsymbol{A}}\left((\forall \underline{e}) \Lambda\left(a_{1}, a_{2}, \ldots, a_{m}, \underline{e}\right)\right)=\mathbf{0}_{\boldsymbol{A}} .
$$

The condition (2) implies that $a_{i}=a_{j}$, for some $i, j$ with $1 \leqslant i<j \leqslant m$. Thus, (1) holds.

The following corollary is a consequence of Theorems 2.5 and 3.3.

Corollary 3.5. Let $m \geqslant 3$ be a natural number. Let $\mathbf{Q}$ be a quasivariety generated by a finite family of algebras with all algebras of cardinality less than $m$. Then $\mathbf{Q}$ has $m$-EDTPM. In fact, $\mathbf{Q}$ has $n$-EDTPM for all $n \geqslant m$.

In other words, the first part of above corollary states that

Every finitely generated quasivariety has $m$-EDTPM for some $m \geqslant 3$.

In view of the above observations, the property of having $m$-EDTPM for some $m$ is essentially weaker than the property of being a finitely generated quasivariety. For example, it follows from Theorem 3.3(2) that every RCD quasivariety $\mathbf{Q}$ has $m$-EDTPM for all $m \geqslant 3$, although $\mathbf{Q}$ need not be finitely generated.

Proof. Assume $\mathbf{Q}$ is generated by a finite set of finite algebras, each of cardinality less than $m$. Suppose $\boldsymbol{A} \in \mathbf{Q}, \Phi \in \boldsymbol{C o n}_{\mathbf{Q}}(\boldsymbol{A})$, and $a_{1}, \ldots, a_{m}$ is a sequence of elements of $A$. By Theorem 2.5, we have that $\bigcap_{1 \leqslant i<j \leqslant m} \Theta_{\mathbf{Q}}^{\boldsymbol{A}}\left(a_{i}, a_{j}\right)=\mathbf{0}_{\boldsymbol{A}}$ and $\bigcap_{1 \leqslant i<j \leqslant m}\left(\Phi+{ }_{\mathbf{Q}} \Theta_{\mathbf{Q}}^{\boldsymbol{A}}\left(a_{i}, a_{j}\right)\right)=\Phi$. Thus,

$$
\Phi+\mathbf{Q} \bigcap_{1 \leqslant i<j \leqslant m} \Theta_{\mathbf{Q}}^{\boldsymbol{A}}\left(a_{i}, a_{j}\right)=\Phi=\bigcap_{1 \leqslant i<j \leqslant m}\left(\Phi+\mathbf{Q} \Theta_{\mathbf{Q}}^{\boldsymbol{A}}\left(a_{i}, a_{j}\right)\right) .
$$

So $\mathbf{Q}$ has $m$-EDTPM by Theorem 3.3.

The following observations supplement Theorem 2.5:

Theorem 3.6. Let $\mathbf{Q}$ be a quasivariety and $m \geqslant 3$ a fixed natural number. The following conditions are equivalent:

(1) $\mathbf{Q}$ is generated by a finite class of algebras each of which has at most $m-1$ elements.

(2) $\mathbf{Q}$ has $m$-EDTPM and $\bigcap_{1 \leqslant i<j \leqslant m} \Theta_{\mathbf{Q}}^{\boldsymbol{F}}\left(x_{i}, x_{j}\right)=\mathbf{0}_{F}$ for any sequence $x_{1}$, $\ldots, x_{m}$ of $m$ different free generators of the free algebra $\boldsymbol{F}:=\boldsymbol{F}_{\mathbf{Q}}(\omega)$.

Proof. (1) $\Rightarrow(2)$ : Assume (1). The first conjunct of (2) follows from Corollary 3.5 and the second conjunct is a particular case of condition (3) of Theorem 2.5.

$(2) \Rightarrow(1)$ : Assume $(2)$. Let $x_{1}, \ldots, x_{m}$ be a sequence of $m$ different free generators of the free algebra $\boldsymbol{F}=\boldsymbol{F}_{\mathbf{Q}}(\omega)$. The fact that $\mathbf{Q}$ has $m$-EDTPM and Theorem 3.3 imply that

$$
\Phi+\mathbf{Q} \bigcap_{1 \leqslant i<j \leqslant m} \Theta_{\mathbf{Q}}^{\boldsymbol{F}}\left(x_{i}, x_{j}\right)=\bigcap_{1 \leqslant i<j \leqslant m}\left(\Phi+{ }_{\mathbf{Q}} \Theta_{\mathbf{Q}}^{\boldsymbol{F}}\left(x_{i}, x_{j}\right)\right),
$$


for any Q-congruence $\Phi$ of $\boldsymbol{F}$. This and the second conjunct of (2) give that

$$
\Phi=\bigcap_{1 \leqslant i<j \leqslant m}\left(\Phi+\mathbf{Q} \Theta_{\mathbf{Q}}^{\boldsymbol{F}}\left(x_{i}, x_{j}\right)\right),
$$

for any $\mathbf{Q}$-congruence $\Phi$ of $\boldsymbol{F}$. So (1) holds by Theorem 2.5.

Note. The second conjunct of (2) is equivalently formulated in terms of the consequence $\mathbf{Q}^{e q \vDash}$ as

(a) $\bigcap_{1 \leqslant i<j \leqslant m} \mathbf{Q}^{e q \vDash}\left(x_{i} \approx x_{j}\right)=\mathbf{Q}^{e q \vDash}(\varnothing)$.

In the presence of the first conjunct, it is equivalent to the condition

(b) $\bigcap_{1 \leqslant i<j \leqslant n} \mathbf{Q}^{e q \vDash}\left(\alpha_{i} \approx \alpha_{j}\right)=\mathbf{Q}^{e q \vDash}(\varnothing)$, for any terms $\alpha_{1}, \alpha_{2}, \ldots, \alpha_{m}$.

Theorem 3.7. Let $m \geqslant 3$ be a natural number. Let $\mathbf{Q}$ be a quasivariety with $m$-EDTPM with respect to a set of equations $\Lambda=\Lambda\left(x_{1}, x_{2}, \ldots, x_{m}, \underline{u}\right)$. The following conditions are equivalent:

(1) $\mathbf{Q}$ is generated (as a quasivariety) by a finite class of algebras of size at most $m-1$.

(2) $\mathrm{Q}$ satisfies the equations $\Lambda\left(x_{1}, x_{2}, \ldots, x_{m}, \underline{u}\right)$.

Proof. $(\forall \underline{u}) \Lambda\left(x_{1}, x_{2}, \ldots, x_{m}, \underline{u}\right)$ is the union of the sets $\Lambda\left(x_{1}, x_{2}, \ldots, x_{m}, \underline{\delta}\right)$ with $\underline{\delta}$ ranging over sequences of terms, each sequence of the length of $\underline{u}$.

$(1) \Rightarrow(2)$ : Assume (1). It follows from Theorem 3.6.(2) that

$$
\bigcap_{1 \leqslant i<j \leqslant m} \Theta_{\mathbf{Q}}^{\boldsymbol{F}}\left(x_{i}, x_{j}\right)=\mathbf{0}_{F},
$$

where $x_{1}, \ldots, x_{m}$ is an arbitrary but fixed sequence of $m$ different free generators of the free algebra $\boldsymbol{F}:=\boldsymbol{F}_{\mathbf{Q}}(\omega)$. As $\mathbf{Q}$ has $m$-EDTPM with respect to $\Lambda$, we therefore get that $\Theta_{\mathbf{Q}}^{\boldsymbol{F}}\left((\forall \underline{e}) \Lambda^{\boldsymbol{F}}\left(x_{1}, x_{2}, \ldots, x_{m}, \underline{e}\right)\right)=\mathbf{0}_{F}$. This is equivalent to $\mathbf{Q}^{e q \vDash}\left((\forall \underline{u}) \Lambda\left(x_{1}, x_{2}, \ldots, x_{m}, \underline{u}\right)\right)=\mathbf{Q}^{e q \vDash}(\varnothing)$. It follows that $\mathbf{Q}^{e q \vDash}\left(\Lambda\left(x_{1}, x_{2}, \ldots, x_{m}, \underline{u}\right)\right)=\mathbf{Q}^{e q \vDash}(\varnothing)$. So (2) holds.

$(2) \Rightarrow(1)$ : (2) implies $\Theta_{\mathbf{Q}}^{\boldsymbol{A}}\left((\forall \underline{e}) \Lambda\left(a_{1}, a_{2}, \ldots, a_{m}, \underline{e}\right)\right)=\mathbf{0}_{\boldsymbol{A}}$, for any $\boldsymbol{A} \in \mathbf{Q}$ and any sequence $a_{1}, \ldots, a_{m}$ of elements of $A$. But $\bigcap_{1 \leqslant i<j \leqslant m} \Theta_{\mathbf{Q}}^{\boldsymbol{A}}\left(a_{i}, a_{j}\right)=$ $\Theta_{\mathbf{Q}}^{\boldsymbol{A}}\left((\forall \underline{e}) \Lambda\left(a_{1}, a_{2}, \ldots, a_{m}, \underline{e}\right)\right)$, because $\mathbf{Q}$ has $m$-EDTPM with respect to $\Lambda$. It follows that $\bigcap_{1 \leqslant i<j \leqslant m} \Theta_{\mathbf{Q}}^{\boldsymbol{A}}\left(a_{i}, a_{j}\right)=\mathbf{0}_{\boldsymbol{A}}$ for any $\boldsymbol{A} \in \mathbf{Q}$ and any sequence $a_{1}, \ldots, a_{m}$ of elements of $A$. This gives (1), by Theorem 2.5.

According to Corollary 2.5, every finitely generated quasivariety $\mathbf{Q}$ has the $m$-EDTPM property for sufficiently large $m$. But, more interestingly, $\mathbf{Q}$ has $m$-EDTPM with respect to a trivial finite set of equations:

Theorem 3.8. Let $m \geqslant 3$ be a natural number. Suppose that $\mathbf{Q}$ is a quasivariety generated by a finite class of algebras each of which has at most $m-1$ elements. Then $\mathbf{Q}$ has $m$-EDTPM with respect to the following finite set of equations:

$$
\Lambda_{m}\left(x_{1}, x_{2}, \ldots, x_{m}\right):=\left\{x_{1} \approx x_{1}, x_{2} \approx x_{2}, \ldots, x_{m} \approx x_{m}\right\} .
$$


Proof. Let $\mathbf{Q}$ be as above. It is clear that for any $\boldsymbol{A} \in \mathbf{Q}$ and $a_{1}, \ldots, a_{m} \in A$, $\Theta_{\mathbf{Q}}^{\boldsymbol{A}}\left(\Lambda_{m}\left(a_{1}, a_{2}, \ldots, a_{m}\right)\right)=\mathbf{0}_{\boldsymbol{A}}$. This means that

$$
\boldsymbol{A} \vDash \wedge \Lambda_{m}\left(x_{1}, x_{2}, \ldots, x_{m}\right)\left[a_{1}, a_{2}, \ldots, a_{m}\right] .
$$

On the other hand, $\bigcap_{1 \leqslant i<j \leqslant m} \Theta_{\mathbf{Q}}^{\boldsymbol{A}}\left(a_{i}, a_{j}\right)=\mathbf{0}_{\boldsymbol{A}}$ by Theorem 2.5.(2). It follows that for any $\boldsymbol{A} \in \mathbf{Q}$ and any $a_{1}, \ldots, a_{m} \in A$, the equivalence

$$
\bigcap_{1 \leqslant i<j \leqslant m} \Theta_{\mathbf{Q}}^{\boldsymbol{A}}\left(a_{i}, a_{j}\right)=\mathbf{0}_{\boldsymbol{A}} \Leftrightarrow \boldsymbol{A} \vDash \wedge \Lambda_{m}\left(x_{1}, x_{2}, \ldots, x_{m}\right)\left[a_{1}, a_{2}, \ldots, a_{m}\right]
$$

is true because both sides are true.

Thus, any algebra $\boldsymbol{A} \in \mathbf{Q}$ satisfies condition (2) of Proposition 3.2. It follows that $\mathbf{Q}$ has $m$-EDTPM with respect to $\Lambda_{m}\left(x_{1}, x_{2}, \ldots, x_{m}\right)$.

The above theorem and the remarks following Definition 2.1 show that if $\mathbf{Q}$ is generated as a quasivariety by a finite class of algebras each of which has at most $m-1$ elements, then $\mathbf{Q}$ has $m$-EDTPM with respect to a set $\Lambda\left(x_{1}, x_{2}, \ldots, x_{m}, \underline{u}\right)$ of equations iff $\Theta_{\mathbf{Q}}^{\boldsymbol{A}}\left((\forall \underline{e}) \Lambda^{\boldsymbol{A}}\left(a_{1}, a_{2}, \ldots, a_{m}, \underline{e}\right)\right)=\mathbf{0}_{\boldsymbol{A}}$ for all $\boldsymbol{A} \in \mathbf{Q}$ and all $a_{1}, a_{2}, \ldots, a_{m} \in \boldsymbol{A}$.

The above proof shows that one cannot expect much from $m$-EDTPM in general while studying specific properties of finitely generated quasivarieties Q - the equations of $\Lambda_{m}\left(x_{1}, x_{2}, \ldots, x_{m}\right)$ from Theorem 3.8 are not conjoined with the intrinsic structure of the algebras of $\mathbf{Q}$. Consequently, the $m$-EDTPM property trivializes for $\mathbf{Q}$. But if one drops the assumption that $\mathbf{Q}$ is finitely generated, the problem becomes less trivial. For example, in the case of quasivarieties endowed with the additive equationally defined commutator the situation differs - there are sets of equations determining $m$-EDTPM whose properties are strictly linked with the commutator $([2,3])$. The definition of the equationally defined commutator makes sense for any quasivariety. The equationally defined commutator coincides with the "standard" commutator in relatively congruence modular (RCM) quasivarieties. For a general account of the commutator for RCM quasivarieties - see [8] or [10]. One may then investigate non-finitely generated quaivarieties possessing $m$-EDTPM with respect to such a commutator-derived set of equations. This issue is not discussed in this paper.

Let $\mathbf{Q}$ be a quasivariety. A quasivariety $\mathbf{Q}^{\prime} \subseteq \mathbf{Q}$ is called a variety relative to $\mathbf{Q}$, or a relative subvariety of $\mathbf{Q}$, if $\mathbf{Q}^{\prime}=\mathbf{V} \cap \mathbf{Q}$ for some variety $\mathbf{V}$ (see e.g., [15, p. 503]). If $\Sigma$ is a base for $\mathbf{Q}$, then every relative subvariety of $\mathbf{Q}$ is of the form $\operatorname{Mod}(\Sigma \cup X)$ for some set of identities $X$. If $\mathbf{Q}^{\prime}$ is a relative subvariety of $\mathbf{Q}$, then the lattice $\boldsymbol{C o n}_{\mathrm{Q}^{\prime}}(\boldsymbol{A})$ is identical with $\boldsymbol{C o n}_{\mathrm{Q}}(\boldsymbol{A})$ for every $\boldsymbol{A} \in \mathbf{Q}^{\prime}$. Moreover $\mathbf{Q}_{\mathrm{RFSI}}^{\prime}=\mathbf{Q}^{\prime} \cap \mathbf{Q}_{\mathrm{RFSI}}$.

Proposition 3.9. Let $\mathbf{Q}^{\prime}$ be a relative subvariety of $\mathbf{Q}$.

(1) If $\mathbf{Q}$ has $m$-EDTPM with respect to a set of equations $\Lambda\left(x_{1}, x_{2}, \ldots, x_{m}, \underline{u}\right)$, then so has $\mathbf{Q}^{\prime}$.

(2) If $\mathbf{Q}$ is finitely generated generated by a finite class of algebras each of which has at most $m-1$ elements, then so is $\mathbf{Q}^{\prime}$. 
Proof. (1): This is immediate from Definition 3.1 and the above remarks.

(2): This is immediate from Theorem 2.5.(2).

Proposition 3.10. Every finitely generated quasivariety $\mathbf{Q}$ has only finitely many relative subvarieties and each of them is finitely based relative to $\mathbf{Q}$.

Proof. Assume $\mathbf{Q}=\boldsymbol{S P}(\mathbf{K})$ for some finite class of finite algebras. Then

$$
\mathrm{Q}_{\mathrm{RFSI}} \subseteq S(\mathbf{K}),
$$

(see e.g., [4, Lemma 1.5]).

If $\mathbf{Q}^{\prime} \subseteq \mathbf{Q}$ is a relative subvariety of $\mathbf{Q}$, then $\mathbf{Q}_{\mathrm{RFSI}}^{\prime}=\mathbf{Q}^{\prime} \cap \mathbf{Q}_{\mathrm{RFSI}} \subseteq$ $\mathrm{Q}_{\mathrm{RFSI}} \subseteq \boldsymbol{S}(\mathbf{K})$ by $(*)$. As the set $\boldsymbol{S}(\mathbf{K})$ is finite and every quasivariety is determined by its relatively subdirectly irreducible algebras, it follows that there are only finitely many quasivarieties $\mathbf{R}$ for which $\mathbf{R}_{\mathrm{RFSI}} \subseteq \boldsymbol{S}(\mathbf{K})$. In particular, there are only finitely many relative subvarieties of $\mathbf{Q}$.

As to the second part, it suffices to show, that if $\Sigma$ is a fixed base for $\mathbf{Q}$, then every relative subvariety of $\mathbf{Q}$ is axiomatized by $\Sigma \cup X$ for some finite set of identities $X$. Suppose not, i.e., there exists an infinite set of equations $X$ such that the relative subvariety $\operatorname{Mod}(\Sigma \cup X)$ is not axiomatized by $\Sigma \cup X_{f}$, for all finite $X_{f} \subset X$. A straightforward argument shows that there exists a sequence of finite sets of equations $X_{0}, X_{1}, \ldots, X_{n}, \ldots$ such that $X=\bigcup_{n \in \omega} X_{n}$ and the classes $\operatorname{Mod}\left(\Sigma \cup X_{n}\right)$, for $n \in \omega$, form a strictly ascending chain. As each of the classes $\operatorname{Mod}\left(\Sigma \cup X_{n}\right)$ is a relative subvariety of $\mathbf{Q}$, this implies that $\mathbf{Q}$ has infinitely many relative subvarieties, a contradiction.

\section{Finitely based quasivarieties and $m$-EDTPM}

There is an ample literature concerning finitely based varieties and quasivarieties-see e.g., [4, 6, 11, 12, 14, 15, 16, 17]. Every finitely generated RCD quasivariety of finite signature is finitely based [15]. An analogous problem for finitely generated RCM quasivarieties appears to be open.

A quasivariety $\mathbf{Q}$ has the Weak Extension Property if for any $\boldsymbol{A} \in \mathbf{Q}$ and any $a, b, c, d \in \mathbf{Q}, \Theta(a, b) \cap \Theta(c, d)=\mathbf{0}_{\boldsymbol{A}}$ implies $\Theta_{\mathbf{Q}}(a, b) \cap \Theta_{\mathbf{Q}}(c, d)=\mathbf{0}_{\boldsymbol{A}}$. This notion is discussed in [6], where suitable examples are provided. Every RCM quasivariety has the Weak Extension Property.

Theorem 4.1. Let $m \geqslant 3$ be a positive integer. Let $\mathbf{Q}$ be a quasivariety of algebras of finite type $\tau$ generated by a finite family of algebras, each of cardinality $\leqslant m-1$. Let us moreover assume that $\mathbf{Q}$ has the Weak Extension Property. The following conditions are equivalent:

(1) $\mathbf{Q}$ is finitely based.

(2) There exist a finitely based quasivariety $\mathbf{Q}^{\prime}$ and a finite set of equations $\Lambda\left(x_{1}, x_{2}, \ldots, x_{m}, \underline{u}\right)$ in $m$ variables (possibly with parameters) with $\mathbf{Q} \subseteq \mathbf{Q}^{\prime}$ and both $\mathbf{Q}$ and $\mathbf{Q}^{\prime}$ having $m$-EDTPM with respect to $\Lambda\left(x_{1}, x_{2}, \ldots, x_{m}, \underline{u}\right)$. 
The theorem asserts that in order to show that $\mathbf{Q}$ is finitely based, it is necessary and sufficient to find a finite set of equations $\Lambda\left(x_{1}, x_{2}, \ldots, x_{m}, \underline{u}\right)$ and a finitely based quasivariety $\mathbf{Q}^{\prime} \supseteq \mathbf{Q}$ such that both $\mathbf{Q}$ and $\mathbf{Q}^{\prime}$ have $m$-EDTPM with respect to $\Lambda$.

Some consequences of the theorem will be presented in another paper.

Proof. (1) $\Rightarrow(2)$ : Assume (1). In view of Theorem 3.8, $\mathbf{Q}$ has $m$-EDTPM with respect to the trivial set of equations,

$$
\Lambda\left(x_{1}, x_{2}, \ldots, x_{m}\right):=\left\{x_{1} \approx x_{1}, x_{2} \approx x_{2}, \ldots, x_{m} \approx x_{m}\right\} .
$$

It suffices to take $\mathbf{Q}^{\prime}:=\mathbf{Q}$.

$(2) \Rightarrow(1)$ : Suppose $\mathbf{Q}^{\prime}$ and $\Lambda\left(x_{1}, x_{2}, \ldots, x_{m}, \underline{u}\right)$ satisfy $(2)$. We define

$$
\mathbf{Q}_{\Lambda}^{\prime}:=\left\{\boldsymbol{A} \in \mathbf{Q}^{\prime}: \boldsymbol{A} \text { universally satisfies } \Lambda\left(x_{1}, x_{2}, \ldots, x_{m}, \underline{u}\right)\right\} .
$$

The quasivariety $\mathbf{Q}_{\Lambda}^{\prime}$ is a relative subvariety of $\mathbf{Q}^{\prime}$. If $\Sigma$ is a finite base for $\mathbf{Q}^{\prime}$ ( such a base exists because $\mathbf{Q}^{\prime}$ is finitely based), then $\mathbf{Q}_{\Lambda}^{\prime}=\operatorname{Mod}(\Sigma \cup \Lambda)$. It follows that $\mathbf{Q}^{\prime}{ }_{\Lambda}$ is also finitely based.

As $\mathbf{Q}_{\Lambda}^{\prime} \subseteq \mathbf{Q}^{\prime}$ is a relative subvariety, the lattice of $\mathbf{Q}^{\prime}{ }_{\Lambda}$-congruences is identical with the lattice of $\mathbf{Q}^{\prime}$-congruences for any algebra $\boldsymbol{A} \in \mathbf{Q}^{\prime}{ }_{\Lambda}$. This implies, by Proposition 3.9.(1), that $\mathbf{Q}^{\prime}{ }_{\Lambda}$ has $m$-EDTPM with respect to $\Lambda$ as well. But in view of Theorem 3.7, as $\mathbf{Q}^{\prime}{ }_{\Lambda}$ satisfies $\Lambda, \mathbf{Q}^{\prime}{ }_{\Lambda}$ is a finitely generated quasivariety (generated by a family of algebras, each algebra of cardinality $\leqslant m-1)$.

On the other hand, the fact that $\mathbf{Q}$ is generated by a finite family of algebras, each of cardinality $\leqslant m-1$, the fact that $\mathbf{Q}$ has $m$-EDTPM with respect to $\Lambda\left(x_{1}, x_{2}, \ldots, x_{m}, \underline{u}\right)$, and Theorem 3.7 imply that $\mathbf{Q}$ satisfies the equations of $\Lambda\left(x_{1}, x_{2}, \ldots, x_{m}, \underline{u}\right)$. As $\mathbf{Q} \subseteq \mathbf{Q}^{\prime}$, it follows that $\mathbf{Q} \subseteq \mathbf{Q}^{\prime}{ }_{\Lambda}$. Thus, $\mathbf{Q}$ is included in the finitely generated and finitely based quasivariety $\mathbf{Q}_{\Lambda}^{\prime}$.

We now apply Theorem 5 of [6].

Suppose $\mathbf{K}$ is a quasivariety of finite signature satisfying the Weak Extension Property. If $\mathbf{K}$ is included in a finitely generated quasivariety $\mathbf{L}$, then $\mathbf{K}$ is finitely axiomatizable relative to $\mathbf{L}$.

As $\mathbf{Q}$ is a quasivariety of finite signature with the Weak Extension Property included in the finitely generated quasivariety $\mathbf{Q}^{\prime}{ }_{\Lambda}$, Theorem 5 implies that $\mathbf{Q}$ is finitely axiomatizable relative to $\mathbf{Q}^{\prime}{ }_{\Lambda}$. As $\mathbf{Q}^{\prime}{ }_{\Lambda}$ itself is finitely axiomatizable, it follows that $\mathbf{Q}$ is finitely axiomatizable.

This concludes the proof of the theorem.

Let $m$ be a positive integer. A quasivariety $\mathbf{Q}$ is residually $<m$ if for every algebra $\boldsymbol{A} \in \mathbf{Q}$ and each pair of distinct elements $a$ and $b$ in $A$ there is an algebra $\boldsymbol{B} \in \mathbf{Q}$ of cardinality less than $m$ and a homomorphism $h: \boldsymbol{A} \rightarrow \boldsymbol{B}$ such that $h(a) \neq h(b)$. Q has a finite residual bound if there is $m<\omega$ such that $\mathbf{Q}$ is residually $<m$ (see [13]).

Note 4.2. In light of the above definition and Theorem 2.5, for any $m \geqslant 3$ and any quasivariety $\mathbf{Q}$ of finite type, the following conditions are equivalent: 
(1) $\mathbf{Q}$ is residually $<m$.

(2) All algebras in $\mathbf{Q}_{\text {RFSI }}$ have cardinality less than $m$.

(3) $\mathbf{Q}=\boldsymbol{S P}(\mathbf{K})$ for a finite class $\mathbf{K}$ of algebras where the algebras in $\mathbf{K}$ have size $<m$.

(4) For any algebra $\boldsymbol{A} \in \mathbf{Q}$ and any sequence $a_{1}, \ldots, a_{m}$ of elements of $A$ of length $m$, it is the case that $\bigcap_{1 \leqslant i<j \leqslant m} \Theta_{\mathbf{Q}}^{\boldsymbol{A}}\left(a_{i}, a_{j}\right)=\mathbf{0}_{\boldsymbol{A}}$.

(It is clear that (2) implies (3). Assuming (3), we get that $\mathrm{Q}_{\mathrm{RFSI}} \subseteq \boldsymbol{S}(K)$ (see the proof of Proposition 3.10). Hence, (2) follows.)

Varieties with $m$-EDTPM. A variety $\mathbf{V}$ is finitely generated if it is of the form $\boldsymbol{H} \boldsymbol{S P}(\mathbf{K})$ for some finite set $\mathbf{K}$ of finite algebras (or, equivalently, $\mathbf{V}=$ $\boldsymbol{H} \boldsymbol{S P}(\boldsymbol{A})$ for some finite algebra $\boldsymbol{A})$.

The next observation shows that finitely generated varieties with $m$-EDTPM are always finitely generated as quasivarieties. This phenomenon has been well recognized for finitely generated CD varieties, where it is a consequence of Jónsson's Lemma.

Theorem 4.3. Let $m \geqslant 3$ be a positive integer. Let $\mathbf{K}$ be a finite set of finite algebras of finite type such that all algebras in $\mathbf{K}$ are of size at most $m-1$. The following conditions are equivalent:

(1) The variety $\boldsymbol{H} \boldsymbol{S P}(\mathbf{K})$ has $m$-EDTPM.

(2) $\boldsymbol{H} \boldsymbol{S P}(\mathbf{K})$ has $m$-EDTPM with respect to the trivial set of equations, $\Lambda_{m}\left(x_{1}, x_{2}, \ldots, x_{m}\right)=\left\{x_{1} \approx x_{1}, x_{2} \approx x_{2}, \ldots, x_{m} \approx x_{m}\right\}$.

(3) $\boldsymbol{H} \boldsymbol{S P}(\mathbf{K})$ is residually $<m$.

The proof of the theorem is based on the following lemma (cf. implication $(1) \Rightarrow(2)$ of Theorem 2.5):

Lemma 4.4. Let $\mathbf{K}$ be a finite set of finite algebras such that all algebras in $\mathbf{K}$ are of cardinality at most $m-1$, where $m \geqslant 3$. Suppose that the variety $\boldsymbol{H S P}(\mathbf{K})$ has $m$-EDTPM. Then for any $\boldsymbol{A} \in \boldsymbol{H S P}(\mathbf{K})$, and any sequence $a_{1}, \ldots, a_{m}$ of elements of $A$ of length $m$,

$$
\bigcap_{1 \leqslant i<j \leqslant m} \Theta^{\boldsymbol{A}}\left(a_{i}, a_{j}\right)=\mathbf{0}_{\boldsymbol{A}} .
$$

Proof of the lemma. Suppose $\boldsymbol{A} \in \boldsymbol{H} \boldsymbol{S P}(\mathbf{K})$ and let $a_{1}, \ldots, a_{m}$ be a sequence of elements of $A . \boldsymbol{A}$ is of the form $\boldsymbol{C} / \Phi$, where $\boldsymbol{C} \in \boldsymbol{S P}(\mathbf{K})$. Without loss of generality, we may assume that $\boldsymbol{C} \subseteq \Pi_{t \in T} \boldsymbol{A}_{t}$, where $\boldsymbol{A}_{t} \in \mathbf{K}$, and hence $\left|\boldsymbol{A}_{t}\right| \leqslant m-1$ for all $t \in T$. Let $\Phi_{t}$ be the restriction of $\operatorname{ker}\left(\pi_{t}\right)$ to $\boldsymbol{C}$, for all $t \in T$. Then $\left|\boldsymbol{C} / \Phi_{t}\right| \leqslant m-1$ for all $t \in T$, and $\bigcap_{t \in T} \Phi_{t}=\mathbf{0}_{\boldsymbol{C}}$. There exists $c_{1}, \ldots, c_{m} \in C$ such that $a_{i}=c_{i} / \Phi$ for $i=1,2, \ldots, m$. For each $t \in T$, there exist $i$ and $j$ with $i<j$ such that $\left\langle c_{i}, c_{j}\right\rangle \in \Phi_{t}$ because $\Phi_{t}$ has index at most $m-1$. It follows that $\bigcap_{1 \leqslant i<j \leqslant m} \Theta^{C}\left(c_{i}, c_{j}\right) \subseteq \Phi_{t}$, for all $t \in T$. Consequently, $\mathbf{0}_{\boldsymbol{C}} \subseteq \bigcap_{1 \leqslant i<j \leqslant m} \Theta^{\boldsymbol{C}}\left(c_{i}, c_{j}\right) \subseteq \bigcap_{t \in T} \Phi_{t}=\mathbf{0}_{\boldsymbol{C}}$, and hence

$$
\mathbf{0}_{\boldsymbol{C}}=\bigcap_{1 \leqslant i<j \leqslant m} \Theta^{\boldsymbol{C}}\left(c_{i}, c_{j}\right) .
$$


Let $h: \boldsymbol{C} \rightarrow \boldsymbol{A}$ be the canonical homomorphism. The above equality, $m$ EDTPM, and $\operatorname{ker}(h)=\Phi$ give that

$$
\begin{aligned}
\Phi & =h^{-1}\left(\mathbf{0}_{\boldsymbol{A}}\right) \subseteq h^{-1}\left(\bigcap_{1 \leqslant i<j \leqslant m} \Theta^{\boldsymbol{A}}\left(a_{i}, a_{j}\right)\right)=\bigcap_{1 \leqslant i<j \leqslant m} h^{-1}\left(\Theta^{\boldsymbol{A}}\left(a_{i}, a_{j}\right)\right) \\
& =\bigcap_{1 \leqslant i<j \leqslant m}\left(\Phi+\Theta^{\boldsymbol{A}}\left(c_{i}, c_{j}\right)\right)=\Phi+\bigcap_{1 \leqslant i<j \leqslant m} \Theta^{\boldsymbol{A}}\left(c_{i}, c_{j}\right)=\Phi .
\end{aligned}
$$

It follows that $h^{-1}\left(\mathbf{0}_{\boldsymbol{A}}\right)=h^{-1}\left(\bigcap_{1 \leqslant i<j \leqslant m} \Theta^{\boldsymbol{A}}\left(a_{i}, a_{j}\right)\right)$. Hence,

$$
\mathbf{0}_{\boldsymbol{A}}=\bigcap_{1 \leqslant i<j \leqslant m} \Theta^{\boldsymbol{A}}\left(a_{i}, a_{j}\right) .
$$

Proof of Theorem 4.3. (2) $\Rightarrow(1)$ : This is trivial.

$(1) \Rightarrow(3)$ : We assume (1). Applying the above lemma and Note 4.2, we get that $\boldsymbol{H S P}(\mathbf{K})$ is residually $<m$.

$(3) \Rightarrow(2)$ : We assume (3). According to Note 4.2 , there exists a finite set $\mathbf{K}^{\prime} \subseteq \boldsymbol{H S P}(\mathbf{K})$, with all algebras in $\mathbf{K}^{\prime}$ of size $\leqslant m-1$, such that $\boldsymbol{H} \boldsymbol{S P}(\mathbf{K})=$ $\boldsymbol{S P}\left(\mathbf{K}^{\prime}\right)$. Theorem 3.8 implies that $\boldsymbol{H S P}(\mathbf{K})$ has $m$-EDTPM with respect to the trivial set of equations $\Lambda\left(x_{1}, x_{2}, \ldots, x_{m}\right)$. So $(2)$ holds.

This concludes the proof of the theorem.

As Keith Kearnes pointed out, $m$-EDTPM is not a Mal'cev property for any $m \geqslant 3$. Indeed, the variety of sets has 3-EDTPM, so if the class of 3-EDTPM varieties were definable by a Mal'cev condition, then every variety would have 3-EDTPM.

Every RCD quasivariety has $m$-EDTPM, for all $m \geqslant 3$. This fact does not extend to (non-finitely generated) RCM quasivarieties.

Let $m \geqslant 3$ be a positive integer. Let $\boldsymbol{A}$ be a finite algebra of cardinality at most $m-1$ such that the variety $\boldsymbol{H} \boldsymbol{S P}(\boldsymbol{A})$ is congruence-modular and not residually finite. ( $\boldsymbol{A}$ may be any finite group which possesses a nonabelian Sylow subgroup - see e.g., $[7, \operatorname{Section} 5]) . \boldsymbol{H} \boldsymbol{S P}(\boldsymbol{A})$ is generated as a quasivariety by no finite set of finite algebras (because otherwise the class $\boldsymbol{H} \boldsymbol{S P}(\boldsymbol{A})_{\text {FSI }}$ would consist of finitely many finite algebras and $\boldsymbol{H} \boldsymbol{S P}(\boldsymbol{A})$ would be residually finite). As $\boldsymbol{H} \boldsymbol{S P}(\boldsymbol{A})$ is not residually $<m$, it fails to have $m$-EDTPM by Theorem 4.3.

Acknowledgment. The author is indebted to Keith Kearnes and the anonymous referee for many comments that have improved the presentation of the article.

\section{REFERENCES}

[1] Czelakowski, J.: Protoalgebraic Logics. Kluwer, Dordrecht (2001)

[2] Czelakowski, J.: General theory of the commutator for deductive systems. Part I. Basic facts. Studia Logica 83, 183-214 (2006)

[3] Czelakowski, J.: The Equationally Defined Commutator (manuscript) (2011)

[4] Czelakowski, J., Dziobiak, W.: Congruence distributive quasivarieties whose subdirectly irreducible members form a universal class. Algebra Universalis 27, 128-149 (1990) 
[5] Czelakowski, J., Dziobiak, W.: The parameterized local deduction theorem for quasivarieties of algebras and its applications. Algebra Universalis 35, 373-419 (1996)

[6] Dziobiak, W., Maróti, M., McKenzie, R., Nurakunov, A.: The weak extension property and finite axiomatizability for quasivarieties. Fund. Math. 202, 199-222 (2009)

[7] Freese, R., McKenzie, R.: Residually small varieties with modular congruence lattices. Trans. Amer. Math. Soc. 264, 419-430 (1981)

[8] Freese, R., McKenzie, R.: Commutator Theory for Congruence Modular Varieties. London Math. Soc. Lecture Note Series, vol. 125, Cambridge University Press, Cambridge (1987)

[9] Gorbunov, V.: A characterization of residually small quasivarieties. Dokl. Akad. Nauk SSSR 275, 204-207 (1984) (Russian)

[10] Kearnes, K., McKenzie, R.: Commutator theory for relatively modular quasivarieties. Trans. Amer. Math. Soc. 331, 465-502 (1992)

[11] Maróti, M., McKenzie, R.: Finite basis problems and results for quasivarieties. Studia Logica 78, 293-320 (2004)

[12] McKenzie, R.: Finite equational bases for congruence modular varieties. Algebra Universalis 24, 224-250 (1987)

[13] McKenzie, R., McNulty, G., Willard, R.: Computational recognition of properties of finite algebras (online pdf view)

[14] Nurakunov, A.: Quasi-identities of congruence-distributive quasivarieties of algebras. Siberian Math. J. 42, 108-118 (2001)

[15] Pigozzi, D.: Finite basis theorems for relatively congruence-distributive quasivarieties. Trans. Amer. Math. Soc. 310, 499-533 (1988)

[16] Willard, R.: A finite basis theorem for residually finite, congruence meet-semidistributive varieties. J. Symbolic Logic 65, 187-200 (2000)

[17] Willard, R.: Extending Baker's theorem. Algebra Universalis 45, 335-344 (2001)

\section{JANUSZ CZELAKOWSKI}

Opole University, Institute of Mathematics and Informatics, Oleska 48, 45-251 Opole, Poland

e-mail: jczel@math.uni.opole.pl

Open Access This article is distributed under the terms of the Creative Commons Attribution License which permits any use, distribution, and reproduction in any medium, provided the original author(s) and the source are credited. 